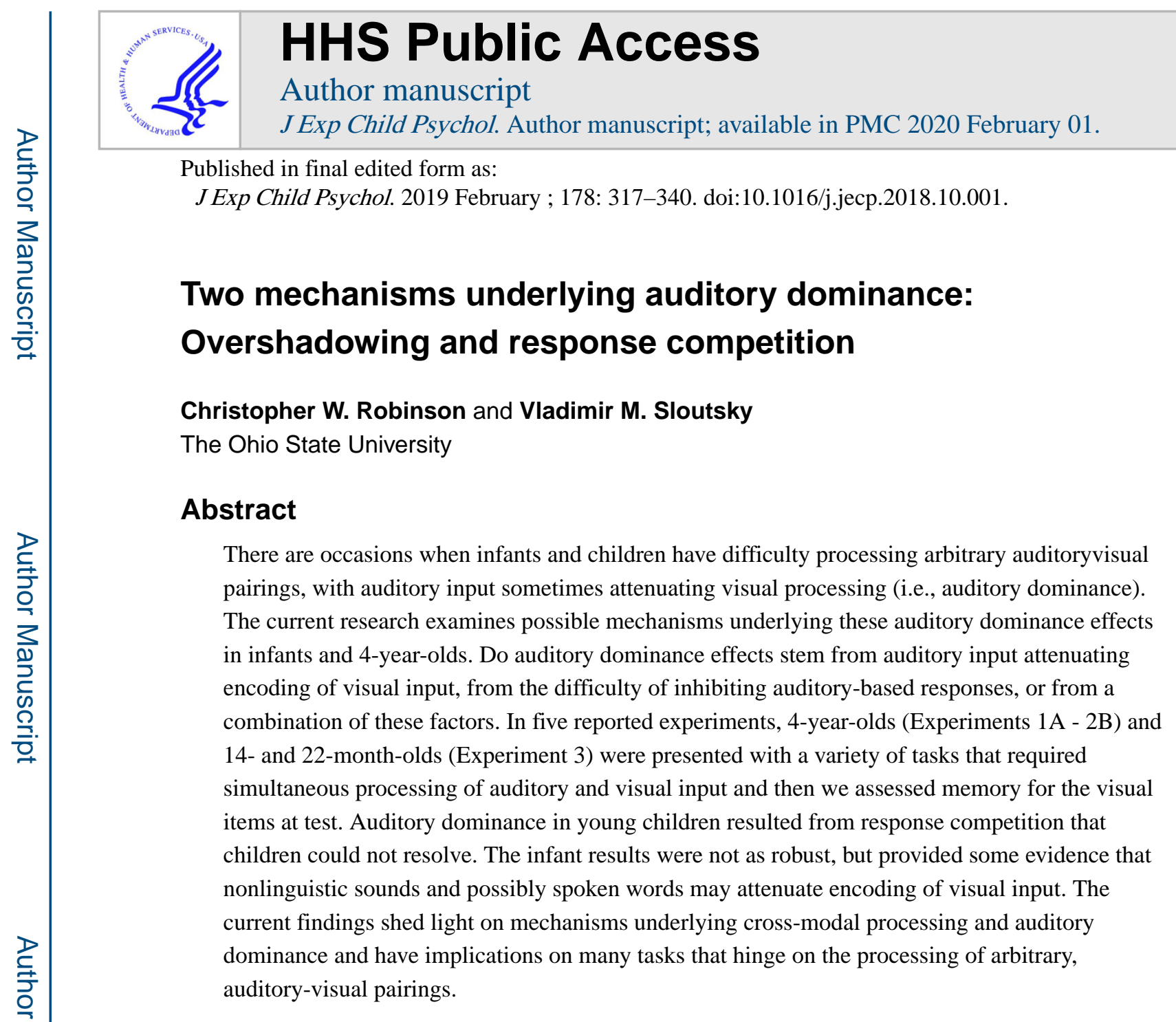

The ability to process simultaneously presented auditory and visual information is a necessary component underlying many cognitive tasks. For example, word learning, lexical extension, and many aspects of category learning hinge on the ability to process simultaneously presented auditory and visual information. While this ability is often taken for granted, there are many occasions when infants and young children process either auditory or visual input, but not both. The reported research aims at understanding the mechanisms underlying these effects across development, with Experiment 1 examining how children process simultaneously presented auditory and visual information, and Experiments 2 and 3 examining how a word learning context affects processing of visual information.

Please address correspondence to Christopher W. Robinson, Department of Psychology, The Ohio State University Newark, 1179 University Drive, Newark, Ohio 43055; Telephone: (740) 755-7843; robinson.777@ osu.edu.

Publisher's Disclaimer: This is a PDF file of an unedited manuscript that has been accepted for publication. As a service to our customers we are providing this early version of the manuscript. The manuscript will undergo copyediting, typesetting, and review of the resulting proof before it is published in its final citable form. Please note that during the production process errors may be discovered which could affect the content, and all legal disclaimers that apply to the journal pertain. 


\section{Cross-modal processing}

Research examining cross-modal processing has documented several important findings.

First, some conditions, reveal cross-modal facilitation, with cross-modal presentation facilitating the processing of amodal relations. For example, when the to-be-learned information (e.g., rhythm, tempo, etc.) is presented to multiple modalities, infants are more likely to learn this information than when the same information is only presented to a single modality (Bahrick, Flom, \& Lickliter, 2002; Bahrick \& Lickliter, 2000), and adults are often faster at detecting and identifying a target when the target is presented cross-modally than to a single modality (Colonius \& Diederich, 2006; Fort, Delpuech, Pernier, \& Giard, 2002; Giard \& Peronnet, 1999; Miller, 1982; Sinnett, Soto-Faraco, \& Spence, 2008). At the same time, cross-modal presentation can also result in modality dominance, with presentation of cross-modal stimuli attenuating processing of modality-specific components. For example, if infants and young children are required to learn modality-specific information (e.g., the shape of an object, the sound the object makes, etc.), then cross-modal presentation may interfere with such learning. Under some conditions, the visual modality wins the competition, and under other conditions, the auditory modality wins the competition (see Robinson \& Sloutsky, 2010a; Sinnett, Spence, \& Soto-Faraco, 2007; Spence, 2009; Spence, Parise, \& Chen, 2012, for reviews).

One interesting component of these modality dominance effects is that they are often asymmetrical in nature, with multisensory presentation (e.g., simultaneously presenting pictures and sounds) attenuating processing in one sensory modality, while having little to no effect on processing in the other modality. Although modality dominance effects are well documented, mechanisms underlying these effects (including their direction and magnitude) are poorly understood. For example, it has been argued that visual dominance in adults may stem from: (1) the visual component of an auditory-visual compound being perceived first (Koppen \& Spence, 2007; Rutschmann \& Link, 1964), (2) a visual response bias to compensate for the poor alerting abilities of visual input (Posner, Nissen, \& Klein, 1976), (3) using spatial tasks which are better suited for the visual modality (Welch \& Warren, 1980), and/or (4) connections between sensory systems being inhibitory in nature (Desimone \& Duncan 1995; Duncan, 1996; Spence et al., 2012). According to the intersensory inhibition account, vision should be more likely to dominate and modulate processing in other modalities because approximately $50 \%$ of the brain is dedicated to the visual modality (Sereno et al., 1995). While there is some evidence that auditory input can modulate visual processing in adults, most of the studies supporting auditory interference/dominance use tasks that are temporal in nature (Mishra, Martinez, \& Hillyard, 2008; Mishra, Martinez, Sejnowski, \& Hillyard, 2007; Parker \& Robinson, 2018; Robinson \& Sloutsky, 2013; Shams, Kamitani, \& Shimojo, 2000; 2002), which should be better suited for the auditory modality (Welch \& Warren, 1980).

Studies examining the developmental trajectory of modality dominance often show that modality dominance effects shift from auditory to visual across development (Nava \& Pavani, 2013; Robinson \& Sloutsky; 2004; Sloutsky \& Napolitano, 2003, see also Hirst, Cragg, \& Allen, 2018; Robinson \& Sloutsky, 2010a, for reviews), thus, it is unclear if the mechanisms proposed to account for visual dominance in adults can account for auditory 
dominance effects in young children. For example, given the relative early maturation of the auditory system relative to the visual system (Birnholz \& Benaceraff, 1983; see also Jusczyk, 1998, for a review), it is possible that young children prioritize auditory processing because the sensory system is more developed. Another account that has been put forward to explain auditory dominance posits that sensory modalities compete for attentional resources (Robinson \& Sloutsky, 2010a; see also Duncan, Martens, \& Ward, 1997; Eimer \& Driver, 2000; Eimer, \& van Velzen, 2002; Pavani, Husain, Ládavas, \& Driver, 2004; Sinnett et al., 2007; Wickens, 1984 for related discussions). Given that auditory stimuli are almost always dynamic and transient in nature, it may be adaptive to first allocate attention to this information before it disappears. While this position assumes that auditory stimuli delay or disrupt visual encoding, it is also possible that these interference effects are more pronounced later in the course of processing (e.g., during the decision/response phase). The primary goal of the current research is to distinguish between these two accounts - do auditory stimuli disrupt encoding of visual information or do they win the competition during the response/decision phase?

\section{Auditory Dominance: Empirical Support}

Evidence for auditory dominance effects was first presented in infants (Lewkowicz, 1988a; 1988b) and then was extended to infants and young children using variations of change detection tasks (Napolitano \& Sloutsky, 2004; Robinson \& Sloutsky, 2004; 2010b; Sloutsky \& Napolitano, 2003; Sloutsky \& Robinson, 2008). In Sloutsky and Napolitano's (2003) task, 4-year-olds and adults participated in an immediate recognition task where they were presented with an auditoryvisual target item followed by an auditory-visual test item. Participants had to indicate whether the target and test items were identical or different. Some test items were identical to the target stimulus (i.e., Old Target). Whereas, other test items had a change in the auditory component (i.e., Changed Auditory/Old Visual), the visual component (i.e., Changed Visual/Old Auditory) or both components changed (i.e., Changed Auditory/Changed Visual). If participants encode auditory and visual input then they should respond same on Old Trials and correctly reject the remaining trial types. While adults exhibited evidence of encoding both auditory and visual components, children encoded only the auditory component. More specifically, children accurately reported when the auditory component changed and when both components changed but failed to reject trials when only the visual component changed (i.e., Changed Visual/Old Auditory trials). These findings are noteworthy because: (1) when the visual stimuli were presented unimodally, children had no difficulty discriminating them (2) visual stimuli had no detrimental effect on auditory processing. Therefore, it was concluded that auditory input overshadowed visual input, thus, resulting in auditory dominance effects in young children but not in adults.

To further examine the development of auditory dominance effects, Robinson and Sloutsky (2004) presented the same auditory and visual compounds to infants, children, and adults. In the infant task, 8-, 12-, and 16-month-olds were familiarized to an auditory-visual compound (see Lewkowicz, 1988a; 1988b for a similar procedure). After $75 \mathrm{~s}$ of familiarization, infants were presented with four test trials: Old Target, Changed Auditory/Old Visual, Changed Visual/Old Auditory and Changed Auditory/Changed Visual. Infants increased looking when 
the auditory component changed and when both components changed (compared to Old Target) but often failed to notice when only the visual component changed. Thus, even though infants discriminated the visual images when presented unimodally, they failed to discriminate the same images when paired with auditory input. A comparison across the age groups demonstrates that auditory dominance effects decreased across development: Infant data were consistent with auditory dominance, children switched between auditory and visual dominance, and adults encoded both modalities. This auditory to visual dominance shift, or increased reliance on visual information, is not specifically tied to change detection tasks - these shifts can also be seen in tasks used to examine Colavita visual dominance, Sound Induced Flash Illusion, and McGurk effects (Massaro, 1984; Nava \& Pavani, 2013; see also Hirst, Cragg, \& Allen, 2018, for a review).

Auditory dominance effects are not fixed and modality dominance may depend on stimuli presented in each modality. For example, when auditory stimuli were more familiar than visual stimuli, auditory dominance effects were observed, whereas, when visual stimuli were more familiar than auditory, visual dominance effects were observed (Napolitano \& Sloutsky, 2004). This finding suggests that increased familiarity with auditory and visual input may affect the allocation of attention in cross-modal processing. Of particular interest to the current study is the finding that familiar sounds and speech-like sounds had comparable effects on visual processing in young children as well as in young infants (Napolitano \& Sloutsky, 2004; Robinson \& Sloutsky, 2007a, 2007b; Sloutsky \& Robinson, 2008). Therefore, it was concluded that some of the effects of words on processing visual input may stem from increased familiarity, with human speech being a familiar class of stimuli for young children.

\section{Goals of Current Research}

While auditory dominance effects are well documented in infants and young children, there are at least two possible mechanisms that can account for these effects. First, it is possible that auditory input disrupts the encoding of visual input (i.e., auditory overshadowing). According to this account, children fail to notice that the visual component changed at test because the visual stimuli were never properly encoded. However, it is also possible that children do encode both modalities, with the auditory modality dominating the visual modality when a response is being generated (i.e., response competition). According to the former mechanism, auditory dominance effects occur early in the course of processing (during the encoding phase), whereas, according to the latter mechanism, auditory dominance effects occur late in the course of processing (during the response/decision phase).

The current study attempts to tease these mechanisms apart by systematically manipulating when auditory stimuli are presented. In some of the reported experiments, children were presented with an auditory-visual target stimulus followed by an auditory-visual testing stimulus. Poor visual discrimination could stem from overshadowing, response competition, or from both mechanisms. In other conditions, children were presented with an auditoryvisual target stimulus followed by a unimodal visual test stimulus, and children had to determine if the two visual stimuli are exactly the same or different. Because the auditory 
stimulus was removed from the testing phase, thus removing any competition during the response/decision phase, attenuated visual processing can be attributed to auditory input disrupting the encoding of the visual input. However, if auditory dominance effects disappear, this would suggest that children do encode visual stimulus, however, the auditory stimulus interferes with visual processing during the response/decision phase.

Since the ability to process auditory and visual information underlies many important tasks such as word learning, a second goal of the current study was to assess how a word learning context affects visual processing in infants and children. Previous research has shown that speech-like sounds, words in isolation, and familiar sounds can attenuate visual processing (Napolitano \& Sloutsky, 2004; Sloutsky \& Napolitano, 2003), however, it is unclear how labeling objects affects encoding of those specific objects. Infants often accumulate more overall looking to labeled objects than non-labeled objects or to objects presented in silence, and infants maintain interest in these objects beyond the labeling episode (Althaus \& Plunkett, 2015; Baldwin \& Markman, 1989; Balaban \& Waxman, 1997; Xu, 2002). One possibility is that this increased looking is associated with better or more efficient processing of the visual information, however, it is also possible that increased attention is directed to the auditory modality. The current research contributes to our understanding of these issues by examining infants' and children's memory for these images in a subsequent recognition task. If words in a word learning context also decrease encoding of visual input, then infants and children who hear labels associated with novel objects should have poorer recognition accuracy compared to infants and children who see the same images in silence.

\section{Overview of Current Research}

To address these questions, we conducted five experiments with infants and young children. In Experiments 1A and 1B, young children were presented with an immediate recognition task. In Experiment 1A, stimuli were presented cross-modally, both in the study and the recognition phases, whereas in Experiment 1B, auditory input was removed during the recognition phase. If auditory dominance stems from attenuated encoding, the removal of auditory input at test cannot affect encoding of visual input at the study phase and thus should not affect the accuracy of responding. Alternatively, if auditory dominance effects stem from response competition occurring at the time of testing, then removing auditory input from test should remove the conflict, thus increasing the accuracy of recognition of visual input. Experiment $2 \mathrm{~A}$ and $2 \mathrm{~B}$ used a more naturalistic word learning task to determine if words presented in a referential context have a similar effect on visual processing. Finally, Experiment 3 focused on infants with the goal to better understand the development of the mechanisms underlying auditory dominance.

\section{Experiment 1A}

The current experiment used a variation of the immediate recognition task, which was used with 4- to 5-year-olds in Sloutsky and Napolitano (2003). Children were presented with a cross-modal target stimulus, which was immediately followed by a cross-modal testing stimulus. Children had to respond "same" if the target and test stimuli were identical or respond "different" if the stimuli differed in any way. Experiment 1B was similar to 
Experiment 1A, except that the auditory input was removed at test. Comparison of recognition accuracy across Experiments 1A-1B should elucidate the mechanism underlying auditory dominance. If recognition of visual input is attenuated (compared to the unimodal baseline) in both Experiments $1 \mathrm{~A}$ and $1 \mathrm{~B}$, it is likely that auditory dominance is driven by attenuated encoding of visual input. However, if recognition of visual input is attenuated only in Experiments 1A, this would be indicative of response competition.

\section{Methods}

\section{Participants and Design}

Twenty-three 4-year-olds ( 11 girls and 12 boys, $M=4.47$ years, $S D=.26$ years) participated in this experiment. Children were recruited through local day-care centers located in middleand upper-middle-class suburbs of XXX. The majority of children were Caucasian. The type of auditory stimulus was manipulated across subjects: Ten children heard familiar sounds associated with the visual images and 13 children heard words associated with the visual images ${ }^{1}$.

\section{Stimuli}

The auditory stimuli consisted of 12 familiar sounds (e.g., car horn, telephone ring, etc.) and 12 nonsense labels (e.g., vika, toma, etc.). Familiar nonlinguistic sounds and words were chosen because these stimuli should be more likely to result in auditory dominance than unfamiliar sounds (Napolitano \& Sloutsky, 2004). Familiarity of the nonlinguistic sounds was assessed in Napolitano and Sloutsky (2004: Experiment 3). The nonsense labels were spoken by a female experimenter using child-directed speech, saved as $44.1 \mathrm{kHz}$ wav files, and edited in Cool Edit so they were $1 \mathrm{~s}$ in duration. The sounds and labels were presented at a comfortable level on a Dell Inspiron laptop computer (approximately $68-72 \mathrm{~dB}$ ). The visual stimuli consisted of a subset of novel animal-like creatures that were reported in Sloutsky, Kloos, and Fisher (2007). The same visual images were used in both auditory conditions (see Figure 1 for examples). Visual stimuli were approximately $10 \mathrm{~cm}$ by $10 \mathrm{~cm}$ in size and were presented for $1 \mathrm{~s}$ in duration.

\section{Procedure}

Cross-modal condition.-The experiment consisted of a cross-modal condition and two unimodal conditions. In the cross-modal condition, children's discrimination of the auditory and visual stimuli was assessed when the stimuli were paired together (cross-modal presentation). Prior to the experiment, children were told that they were going to play a matching game where they would be given a picture and a sound/word and then another picture and sound/word. If both the pictures and sounds/words were exactly the same, they were told to say "same". If the sound/word changed, the picture changed, or both the sound/ word and picture changed, they were told to say "different".

${ }^{1}$ There was no effect of stimulus type and stimulus type never interacted with any of the other variables in Experiments $1 \mathrm{~A}$ and $1 \mathrm{~B}$, all $F_{\mathrm{s}}<1.91, p s>.186$, thus we collapsed across stimulus type. 
Six different auditory-visual compound targets were constructed and each target had four different test trial types, which resulted in 24 trials (see Figure 1 for example of trial types). On each trial, the compound target was presented for $1 \mathrm{~s}$, followed by a $1 \mathrm{~s}$ inter-stimulus interval. A test compound was then presented for $1 \mathrm{~s}$. Six of the test trials had the same auditory-visual components as the target (i.e., Same trials). A correct response on these trials was "same". The remaining test trials had a change in either the auditory component (i.e., Different Auditory/Same Visual), the visual component (i.e., Different Visual/Same Auditory) or both components changed (i.e., Both different), with six trials of each trial type. A correct response on these latter three trial types was "different". The 24 cross-modal trials were randomized for each subject.

Unimodal condition.-After completing the 24 cross-modal trials, children immediately moved to the next phase where the same stimuli were presented unimodally and their discrimination was assessed. Prior to participating in the two unimodal conditions, children were told that they were going to play a similar game, however, this time they would only see pictures or hear sounds/words. The auditory and visual stimuli were identical to those presented in the cross-modal phase, thus, making performance in this phase a measure of baseline discrimination. In the auditory unimodal baseline, children were presented with six same trials (e.g., $\mathrm{AUD}_{1} \rightarrow \mathrm{AUD}_{1}$ ) and six new trials (e.g., $\mathrm{AUD}_{1} \rightarrow \mathrm{AUD}_{2}$ ), and trial order was randomized for each child. In the visual unimodal baseline, children were presented with six same trials (e.g., VIS $\rightarrow$ VIS $_{1}$ ) and six new trials (e.g., VIS $_{1} \rightarrow$ VIS $_{2}$ ), and trial order was randomized for each child. Order of auditory and visual unimodal blocks was randomized across participants.

The cross-modal task is more complex than the unimodal task. In the current study, we ran the cross-modal condition first to decrease the likelihood that poor performance in the crossmodal condition resulted from some of the children being fatigued at the end of the study. However, it is important to note that a recent study using a variation of the immediate recognition task randomized order and found auditory dominance in both children and young adults (Barnhart, Rivera, \& Robinson, 2018).

\section{Results and Discussion}

Discrimination accuracy was calculated separately for the unimodal and cross-modal conditions. Perfect discrimination would result if children responded "same" on all same trials (100\% hit rate) and responded "different" on all different trials (0\% False Alarm rate). False Alarms in the unimodal auditory and unimodal visual conditions reflect the proportion of "same" responses on different trials (e.g., $\mathrm{AUD}_{1} \rightarrow \mathrm{AUD}_{2}$ and $\mathrm{VIS}_{1} \rightarrow \mathrm{VIS}_{2}$ ). Auditory False Alarms on cross-modal trials reflect the proportion of "same" responses on Different Auditory/Same Visual trials. Visual False Alarms on cross-modal trials reflect the proportion of "same" responses on Different Visual/Same Auditory trials. The proportion of Hits and False larms across the different conditions are reported in Table 1. We converted the proportion of Hits and False Alarms to A-prime, which is a nonparametric analogue of the d-prime statistic (Snodgrass \& Corwin, 1988). As accuracy increases, A-primes approach 1.0 , with 0.5 reflecting no-discrimination performance. 
A-primes were submitted to a 2 (Tested Modality: Auditory vs. Visual) x 2 (Presentation Mode: Cross-modal vs. Unimodal) repeated measures ANOVA. There was a main effect of Presentation Mode, $F(1,22)=37.11, p<.001, \eta_{\mathrm{p}}^{2}=.63$, with $\mathrm{A}^{\prime}$ being higher in the unimodal condition $(M=.99, S E=.01)$ than in the Cross-modal condition $(M=.85, S E=$. 02). The effect of Modality, $F(1,22)=21.53, p<.001, \eta_{\mathrm{p}}{ }^{2}=.50$, was also significant, with A' being higher in the Auditory modality $(M=.98, S E=.01)$ than the visual modality ( $M$ $=.86, S E=.01)$. There was also a Presentation Mode by Tested Modality interaction, $F(1$, $22)=19.01, p<.001, \eta_{\mathrm{p}}^{2}=.46$ (see Figure 2). The interaction indicates that relative to the unimodal baselines, cross-modal presentation had a greater cost for visual processing, $t(22)$ $=5.43, p<.001, d=1.55$, than for auditory processing, $t(22)=2.52, p=.020, d=.67$. As can be seen in Table 1, decreased visual discrimination in Experiment 1A was driven by increased false alarms on Different visual/Same auditory trials (i.e., saying "same" when only the visual stimulus changed), with participants making significantly more false alarms on these trials than when the same visual stimuli were presented unimodally, $t(22)=6.41, p$ $<.001, d=1.34$.

Auditory dominance was also corroborated at the individual level. We categorized children as auditory responders, visual responders, equal responders, or attenuated responders. Equal responders correctly identified at least 4 out of 6 Different Auditory/Same Visual trials and correctly identified 4 out of 6 Different Visual/Same Auditory trials. Attenuated responders failed to reach this criterion in both modalities. Auditory responders correctly identified at least 4 out of 6 Different Auditory/Same Visual trials but failed to reach this criterion on Different Visual/Same Auditory trials. Visual responders correctly identified at least 4 out of 6 Different Visual/Same Auditory trials but failed to reach this criterion on Different Auditory/Same Visual trials. The number of children broken down by category were submitted to a chi square, which revealed that a significant proportion of children were classified as auditory responders, $\chi^{2}(3, N=23)=19.96, p<.001$, Fourteen children $(61 \%)$ were classified as auditory responders, seven children $(30 \%)$ were classified as equal responders, one child (4\%) was classified as an attenuated responder, and one child (4\%) was classified as a visual responder ${ }^{2}$. It is also important to note that every child correctly rejected 4 out of 6 Different trials when the visual stimuli were presented unimodally, thus, the significant proportion of auditory responders cannot be accounted for by poor discrimination of the visual stimuli. Therefore, Experiment 1A replicated the signature pattern or modality dominance where cross-modal presentation had greater costs on visual processing than auditory processing (Napolitano \& Sloutsky, 2004; Sloutsky \& Napolitano, 2003).

\section{Experiment 1B}

The goal of Experiment 1B was to determine if attenuated processing of visual input in Experiment 1A stemmed from poor encoding of visual input or from response competition. Children in the current experiment were presented with an immediate recognition task where they were presented with an auditory-visual target stimulus. In contrast to Experiment 1A,

\footnotetext{
${ }^{2}$ A similar pattern emerges when using 5 out of 6 as a criterion, with 13 children (57\%) being classified as auditory responders, 6 (26\%) equal responders, $3(13 \%)$ as attenuated responders, and $1(4 \%)$ visual responder.
} 
stimuli were presented unimodally at test, thus removing any competition during the response phase. If auditory dominance effects disappear, this would suggest that response competition underlies auditory dominance effects reported in Experiment 1A.

\section{Method}

Participants

Eighteen 4-year-olds ( 12 girls and 6 boys, $M=4.4$ years, $S D=.30$ years) participated in this experiment. Nine children heard familiar sounds paired with the visual stimuli and nine children heard nonsense labels paired with the same visual stimuli. Demographics and subject recruitment were identical to Experiment 1A.

\section{Stimuli and Procedure}

The auditory and visual stimuli in the unimodal condition were identical to Experiment $1 \mathrm{~A}$. In contrast to Experiment 1A, children in the cross-modal condition of the current experiment were presented with an auditory-visual target stimulus followed by a unimodal testing stimulus (see Figure 3 for an overview of trial types). Half of the trials were auditory trials and half of the trials were visual trials, and order of trials was randomized for each child. Thus, children had no way of knowing whether they were going to be asked about the sound/word or the picture while the target stimulus was presented. On visual test trials children were asked "were those two pictures the same or different" and on auditory test trials they were asked "were those two sounds/words the same or different". The unimodal condition was identical to Experiment 1A.

\section{Results and Discussion}

Mean proportions of Hits and False Alarms are presented in Table 1. As in Experiment 1A, we submitted A-Primes to a 2 (Tested Modality: Auditory vs. Visual) x 2 (Presentation Mode: Cross-modal vs. Unimodal) repeated measures ANOVA. Data are presented in Figure 4. In contrast to Experiment $1 \mathrm{~A}$, the analysis revealed no significant effects or interactions, all $F \mathrm{~s}<1.05, p \mathrm{~s}>.32$. Therefore, while Experiment $1 \mathrm{~A}$ revealed auditory dominance, there were no signs of auditory dominance in Experiment 1B (where auditory input was removed at test). This difference suggests that auditory dominance originates at the time of responding rather than at the time of encoding. In particular, when auditory input triggered response A (say, "Same") and visual input triggers response B (say, "Different"), the response triggered by auditory input won over the response triggered by visual input.

In short, findings of Experiment 1 shed light on the mechanisms underlying auditory dominance effects. While auditory dominance could stem from failure of encoding and/or response competition, Experiments 1A-1B jointly demonstrate that children do in fact encode the visual stimulus, however, they fail to notice or report these changes when images are paired with auditory input. These findings show that auditory dominance effects, at least in the current manipulations, occur relatively late in the course of processing when children are making comparisons between two cross-modal stimuli. These findings have implications on many tasks that require processing of cross-modal stimuli and also have implications on the development of auditory dominance effects. 


\section{Experiment 2A}

In Experiment 2, we were interested in how a word learning context would affect encoding/ memory of the visual referents. This change in focus required substantial change in stimuli and procedure. In Experiments 2A-2B, children were presented with a word learning task in which they heard novel words associated with novel objects. After the word learning phase, participants' recognition of the studied objects were examined and their recognition accuracies were compared to that in the silent baseline condition. In Experiment 2A, words were presented at training and test, whereas in Experiment 2B, words were presented only during training.

Several outcomes could be expected. First, it is well documented that infants and younger children accumulate more looking to visual stimuli when they are presented in a word learning situation than when presented in silence (Balaban \& Waxman, 1997; Baldwin \& Markman, 1989; Xu, 2002). Therefore, it is possible that the word learning scenario will direct children's attention to the referents and facilitate visual processing. According to this account, it is possible that children will be more likely to encode the referents in the word learning condition than in a silent condition. However, because auditory input often results in auditory dominance effects in young children, with familiar sounds and human speech sounds attenuating visual processing (Napolitano \& Sloutsky, 2004; Sloutsky \& Napolitano, 2003), it was hypothesized that a word learning context would also attenuate visual processing. According to this account, children in the silent condition should make finer visual discriminations than children in the word learning condition.

\section{Method}

\section{Participants}

Thirty-one 4-year-olds ( 20 girls and 11 boys, $M=4.51$ years, $S D=0.30$ years) participated in this experiment. Demographics and subject recruitment were identical to Experiment 1A. Children were randomly assigned to one of two conditions: A word learning condition ( $\mathrm{n}=$ 17) or a silent condition $(n=14)$.

\section{Stimuli}

Four nonsense labels (i.e., "ziblet", "flurp", "hune" and "leru") were presented in children in the word learning condition. The words were spoken by a female experimenter in childdirected speech and embedded within a referential context (e.g., "This is a ziblet"). The auditory stimuli were presented to children on a Dell Inspiron laptop computer with Presentation software at a comfortable level (approximately 68-72 dB). Auditory stimuli ranged from 1.4 to $1.8 \mathrm{~s}$ in duration. During the training phase, each nonsense label was associated with a different referent (see Figure 5 for examples of visual stimuli). Each referent was approximately $10 \mathrm{~cm}$ x $10 \mathrm{~cm}$ and was presented centrally on the Dell Inspiron laptop. We increased the discriminability between the referents to eliminate potential interference. As can be seen in the figure, we presented one of the novel creatures from Experiment 1, however, the other referents were perceptually different. During the testing phase, children were presented with three types of trials: Old referents, critical lures, and 
catch trials. Old referents were identical to the stimuli presented during training and critical lures were perceptually similar to the referents. Given the increased delay between training and testing, we also wanted to make sure that children were still attentive during the testing phase, so we also included catch trials, which were perceptually dissimilar from the referents. Critical lures and catchers were also $10 \mathrm{~cm} \times 10 \mathrm{~cm}$ in size.

\section{Procedure}

The procedure consisted of two phases: Training phase and testing phase. Prior to training, children in the word learning condition and in the silent condition were told to pay close attention to the pictures because they were going to be asked about them at a later time. During the training phase, children were presented with the four referents, presented one at a time. Children in the word learning condition heard a word associated with each referent (e.g., "This is a ziblet"). Children in the silent condition heard no auditory input. The durations of the auditory and visual stimuli were equated, ranging from 1.4 to $1.8 \mathrm{~s}$. Stimulus duration was also equated across the word learning and silent conditions. After the presentation of the fourth referent, children immediately moved to the testing phase.

At test children were told that they were going to see some more pictures, and that some of the pictures would be exactly the same as the ones they just saw and some would be different. There were three types of testing trials: Old referents, critical lures, and catchers. On old referent trials, the word-referent pairings were identical to training (i.e., old word/old referent). In the visual baseline condition, the old visual items were presented in silence. On these trials, children in both conditions should respond "same" because the visual stimulus presented at test was identical to one of the referents presented during the training phase. On critical lure trials, the perceptually similar foils were paired with the old words (i.e., old word/new referent) in the word learning condition and were presented in silence in the baseline condition. Children in both conditions should respond "different" because the visual images were new. On catch trials, both the auditory and visual stimuli were new in the word learning condition. A new catch stimulus was presented in silence in the baseline condition. On catch trials children in both conditions should respond "different" because the visual stimuli presented at test differed from the original referents.

\section{Results and Discussion}

All children correctly rejected catcher trials, thus, no child was excluded from the final sample. Mean proportions of Hits on Old Items and False Alarms on Critical Lures are presented in Table 1, and A-primes were submitted to an independent samples $t$ test. Children in the silent condition were better at discriminating referents from critical lures ( $M$ $=.82, S E=.05)$ than children in the word learning condition $(M=.61, S E=.04)$, independent-sample $t, t(29)=3.27, p=.003, d=1.24$. As in Experiment 1A, attenuated visual discrimination in the current experiment resulted from an increase in false alarms when critical lures were paired with old labels at test compared to the unimodal visual condition, $t(29)=3.04, p<.005, d=.97$. Thus, while previous research has demonstrated that nonlinguistic sounds and speech-like sounds attenuate visual processing in young children (Sloutsky \& Napolitano, 2003; Napolitano \& Sloutsky, 2004), the current findings 
demonstrate that these effects are also present in a word learning situation. In particular, children were better at discriminating visual images when they were presented in silence than when the same visual stimuli were presented in a word learning context.

\section{Experiment 2B}

In Experiment 2B children were taught the same novel word-object pairings as in Experiment 2A, however, in contrast to Experiment 2A, visual stimuli were not paired with words during the testing phase. If poor visual discrimination in Experiment $2 \mathrm{~A}$ stems from response competition then effects should attenuate or disappear in Experiment $2 \mathrm{~B}$ because the auditory input was removed from the testing phase, thus, eliminating competition between modalities. At the same time, if auditory input disrupts the encoding of the visual stimulus, then removing the auditory stimulus at test should have little effect on visual discrimination because the visual stimuli were never properly encoded.

\section{Method}

\section{Participants, Stimuli, and Procedure}

Fourteen 4-year-olds ( 7 girls and 7 boys, $M=4.39$ years, $S D=.26$ years) participated in this experiment. Demographics and subject recruitment were identical to previous experiments, however, in contrast to Experiment $2 \mathrm{~A}$, there was only one condition in the current experiment. The stimuli were identical to Experiment 2A. Children were trained on fourword referent pairings and visual images were presented in silence during the testing phase. Discrimination in the current experiment was compared to the silent and word learning conditions of Experiment 2A.

\section{Results and Discussion}

All children correctly classified the catchers as different, thus, no child was excluded from the primary analyses. Hits on Old Items and False Alarms on Critical Lures are presented in Table 1, and A-primes were submitted to an independent samples $t$ test. Recognition accuracy in the current experiment was compared to the word learning condition of Experiment 2A (where words were presented at test). Children were significantly better at discriminating visual stimuli in the current experiment when the words were removed at test $(M=.78, S E=.05)$ than in the word condition of Experiment 2A $(M=.61, S E=.04)$ where words were presented during the training and testing phases, $t(29)=2.67, p=.012, d=.98$. Discrimination accuracy in the current experiment did not differ from the unimodal visual condition of Experiment 2A, $t(26)=0.43, p=.67, d=.14$, however, it is important to note that removing the words at test in Experiment $2 \mathrm{~B}$ marginally decreased the hit rate compared to the visual baseline condition, $t(26)=1.96, p=.059, d=.71$ (see Table 1). Given that the false alarm rate did not differ between Experiment $2 \mathrm{~B}$ and the unimodal visual condition, $t$ $(26)=1.50, p=.145, d=.55$, it appears that the referents were more difficult to recognize without the label. In summary, these findings demonstrate that attenuated visual discrimination is likely to stem from auditory stimuli winning the competition during the response/decision phase, rather than from auditory input disrupting the encoding of the visual stimulus. 


\section{Experiment 3}

The finding that response competition could account for auditory dominance effects raises an important question: Can all auditory dominance effects and, more generally, attenuated visual processing be accounted for by response competition or are there some situations where auditory input interferes with the encoding of visual input? Experiment 3 begins to address this question by examining how auditory input affects visual processing in younger participants.

Recall that tasks examining auditory dominance in infancy often familiarize or habituate 6to 16-month-old infants to an auditory-visual target stimulus and encoding of the auditory and visual stimuli are assessed by examining infants' looking on test trials (Lewkowicz, 1988a; 1988b; Robinson \& Sloutsky, 2004; 2010a; Sloutsky \& Robinson, 2008). Increased looking on test trials when only the visual component changes (i.e., Different visual/Old auditory trials) compared to Old Target (i.e., Old Visual/Old Auditory) is often taken as evidence that infants encoded the visual stimulus. However, the findings of Experiments 1B and $2 \mathrm{~B}$ raise the possibility that infants do in fact encode visual stimuli but do not increase looking on Different visual/Old auditory trials because the visual stimulus elicits a weak novelty response and the auditory stimulus elicits a strong familiarity response (response competition).

Response competition may also explain why auditory stimuli appeared to slow down or delay the onset of visual processing. For example, Robinson and Sloutsky (2007a) presented 14-month-old infants with a continuous familiarization task (see Fantz, 1964; Roder, Bushnell, \& Sasseville, 2000, for similar procedures). Infants in this study were familiarized to two simultaneously presented visual stimuli; one visual stimulus remained unchanged across familiarization (i.e., familiar), whereas, the other visual stimulus changed on every trial (i.e., novel). Infants either heard an auditory stimulus at the onset of each trial or the visual stimuli were presented in isolation. Percent looking to the novel visual stimulus was assessed on every trial, and visual processing speed was inferred by the amount of familiarization needed before infants demonstrated a reliable novelty (or familiarity) preference.

Two findings from the Robinson and Sloutsky (2007a) study are significant for the current study. First, 14-month-olds were faster at processing visual images (i.e. required less exposure to the stimuli before showing a reliable novelty preference) when they were presented in silence than when paired with unfamiliar sounds. However, because sounds were presented on every trial, it unclear if these effects stem from auditory input interfering with the encoding of visual input or from response competition. A second important finding was that unfamiliar auditory stimuli were more likely to slow down visual processing than familiar auditory stimuli (i.e., prefamiliarized sounds and words). This pattern of results differs considerably from the pattern reported in older children. In particular, while increasing the familiarity of the auditory stimulus corresponds with stronger auditory dominance effects in young children (Napolitano \& Sloutsky, 2004), increasing the familiarity of the auditory stimulus weakens auditory dominance in young 8- to 16-monthold infants (Robinson \& Sloutsky, 2007a; 2010b; Sloutsky \& Robinson, 2008). The 
differential effect of familiarity may suggest that different mechanisms are driving auditory dominance effects.

While unfamiliar auditory stimuli appear to have a greater cost for visual processing than familiar auditory stimuli, there is also some evidence that linguistic input (familiar class of auditory stimuli) can attenuate processing of visual information. For example, in Sloutsky and Robinson (2008), 8- and 12-month-olds often failed to discriminate two visual images when paired with linguistic labels, whereas, they ably discriminated the same visual stimuli when presented in silence. However, labels were also presented at test, so it is unclear if these effects stem from overshadowing or response competition.

Labels presented in a referential context (e.g., "Look, a Dax") can also affect performance on higher-order tasks such as categorization and individuation. Most of the research demonstrates that exposure to linguistic labels can have different effects on categorization and individuation than exposure to nonlinguistic sounds (Balaban \& Waxman, 1997; Ferry, Hespos, \& Waxman, 2010; Fulkerson \& Haaf, 2003; Plunkett, Hu, \& Cohen, 2008; Xu, 2002, see also Robinson, Best, Deng, \& Sloutsky, 2012 for a review), however, this could stem from words facilitating categorization and individuation or from nonlinguistic sounds interfering. Only a handful of studies have directly compared performance in the auditory conditions to a silent baseline condition. Consistent with the modality dominance research, compared to a silent baseline condition, nonlinguistic sounds disrupted categorization in 8and 12-month-olds (Robinson \& Sloutsky, 2007b) and disrupted individuation in 8- and 14month-olds (Robinson \& Sloutsky, 2008). Exposure to linguistic labels had weaker interference effects and either did not differ from the silent baseline or interfered with learning (Robinson \& Sloutsky, 2007b; Robinson \& Sloutsky, 2008, but see Althaus \& Westermann, 2016). It is also important to note that categorization and individuation studies often remove the auditory stimulus during the testing phase, which might suggest that auditory stimuli are overshadowing visual input. Thus, while unfamiliar sounds appear to have greater costs for visual processing than familiar sounds such as human speech, there is some evidence suggesting that hearing linguistic labels associated with possible referents might overshadow visual information in 8- to 14-month-old infants. The current experiment examined if some of these effects persist in slightly older infants.

The goal of the current experiment was to determine if response competition could account for attenuated visual processing in younger participants. In the current experiment, 14- and 22-month-olds were taught two auditory-visual pairings. During a subsequent testing phase, encoding of the referents was assessed. As in Experiments 1B and 2B, auditory stimuli were removed from the testing phase, thus, removing competition between auditory and visual modalities. There were three stimulus conditions, which were manipulated between subjects. As in Experiment 2A, some children were taught word-referent pairings during training (word learning condition) and other children heard no auditory input (silent condition). Another group of infants were presented with unfamiliar sound-referent pairings during the training phase. This condition was added because previous research examining effects of auditory input on visual processing in infants has demonstrated that unfamiliar auditory stimuli often elicit stronger interference than familiar sounds and words (Robinson \& Sloutsky, 2007a; 2010b; Sloutsky \& Robinson, 2008). 
The current experiment also extends previous research by examining visual processing speed and individual learning rates. In the current experiment, the training and testing phases were blocked and repeated across the experiment (e.g., Block 1: Training $\rightarrow$ Test, Block 2: Training $\rightarrow$ Test, etc.). On each test trial, children were presented with two visual images and no auditory input was presented. One visual stimulus was one of the trained referents (i.e., familiar stimulus) and the second stimulus changed on every trial (i.e., novel stimulus). Visual processing speed was assessed by the amount of familiarization needed before infants demonstrated a reliable novelty or familiarity preference.

Several possible outcomes can be predicted. First, it is well documented infants accumulate more looking to visual images when presented in a word learning context or when paired with sounds than when images are presented in silence (Balaban \& Waxman, 1997; Baldwin \& Markman, 1989; Roberts, 1995; Xu, 2002). If increased looking to the visual stimulus corresponds with an increase in visual processing, then it is possible that children in the word condition may be faster at learning the visual images than children in the silent and sound conditions. However, it is also possible that increased overall looking stems from increased attention to the auditory modality, with infants looking to the source of the auditory stimulus (e.g., auditory overshadowing). If this is the case, then increased looking may have no effect on visual processing or possibly even slow down visual processing. Finally, it is possible that effects stem from response competition. According to this account, auditory interference effects should not be present in the current experiment because auditory stimuli were removed from the testing phase, thus, removing competition between auditory and visual modalities.

\section{Method}

\section{Participants}

Thirty-seven 14-month-olds ( 17 boys and 20 girls, $M=437$ days, $S D=65$ days) and 4122 month-olds ( 16 boys and 25 girls, $M=675$ days, $S D=62$ days) participated in this experiment. Parents' names were collected from local birth announcements, and contact information was obtained through local directories. All infants had normal vision and hearing, as reported by parents, and a majority of infants were Caucasian. Twenty-six infants heard labels during training, 27 infants heard nonlinguistic sounds during training, and 25 infants heard no auditory input during training. An additional 11 infants were tested but not included in the current experiment due to fussiness.

\section{Apparatus}

Infants were seated on parents' laps approximately $100 \mathrm{~cm}$ away from a $152 \mathrm{~cm}$ x $127 \mathrm{~cm}$ projection screen. A NEC GT2150 LCD projector presented images to the infants and was mounted on the ceiling approximately $30 \mathrm{~cm}$ behind the infant $(130 \mathrm{~cm}$ away from the projection screen). Two Boston Acoustics 380 speakers presented auditory stimuli to infants. These speakers were $76 \mathrm{~cm}$ apart from each other and mounted in the wall at the infant's eye level. The projector and speakers received visual and auditory signals from a Dell Dimension 8200 computer, which was controlled by Presentation software. This computer was also used to record visual fixations. Fixations were recorded online by pressing a button 
on a 10-button USB gamepad when infants were looking at the stimulus and releasing the button when infants looked away from the stimulus.

\section{Stimuli}

The auditory stimuli consisted of two nonsense words and two unfamiliar sounds. The nonsense words (i.e., "ziblet" and "leru") were produced by a female experimenter using infant-directed speech and were embedded in a linguistic context (e.g., "This is a ziblet"). The nonlinguistic sounds were created by using preset functions in Cool Edit 2000 (i.e., bell and out of control), which were synthesized sounds that changed in pitch and amplitude over time. Note that auditory stimulus condition was manipulated between subjects, so infants either heard speech during training, non-linguistic sounds during training, or they heard no auditory stimuli (baseline). The stimulus onset and duration of the sounds corresponded with the stimulus onset and duration in the word condition. All auditory stimuli were recorded as $44.1 \mathrm{kHz}$ wav files and were presented at a comfortable level to infants (approximately 68 $72 \mathrm{~dB})$.

The visual stimuli consisted of two referents and 24 critical lures (see Figure 6 for examples). During the training phase, the two nonsense words or two unfamiliar sounds cooccurred with two visual images (i.e., referents). The referents were approximately $25 \mathrm{~cm}$ by $35 \mathrm{~cm}$ in size and were projected centrally on the projection screen. In the testing phase, infants were simultaneously presented with one of the trained referents (familiar stimulus) and a critical lure. The horizontal distance between referent and foil was $70 \mathrm{~cm}$. As can be seen in Figure 6, the critical lures shared the same overall shape as the referents but varied in color and texture. To ensure that critical lures remained novel, 24 different stimuli were presented throughout the experiment, one critical lure for each test trial.

\section{Procedure}

The procedure consisted of a training phase and a testing phase, which were blocked and repeated six times throughout the experiment (i.e., Block 1: Training $\rightarrow$ Test, Block 2: Training $\rightarrow$ Test, etc.). Each training phase consisted of two trials. Prior to each training trial, infants were presented with a fixation stimulus (i.e., red blinking circle with beeping sound). When infants looked at the fixation stimulus, it disappeared and was replaced by one of the two referents. Infants in the auditory conditions heard the same word or the same sound associated with the referent three times during the trial (e.g., "This is a ziblet.", "Do you see the ziblet?", "Look, a ziblet."). Infants in the silent condition heard no auditory input associated with the referent. The referent then disappeared and infants were then presented with a fixation stimulus, followed by the second referent. The training trials in all three conditions were $8 \mathrm{~s}$ in duration, and the presentation order of the referents was randomized within each training block. The experimenter recorded infants' visual fixations on each training trial by pressing a button on the USB gamepad when infants were looking at the stimulus and by releasing the button when infants looked away.

Immediately after the training phase, infants moved to the testing phase, which consisted of four test trials. As in the training phase, a fixation stimulus was used to engage the infant prior to each test trial. When infants looked at the fixation stimulus, it disappeared and 
infants were presented with a test trial, which consisted of the simultaneous presentation of one of the trained referents (familiar stimulus) and a critical lure (novel stimulus). Each test trial was $8 \mathrm{~s}$ in duration and no auditory input was provided on test trials. The two referents were each presented twice during the testing block, and the left-right location of the referent was counter-balanced within each testing block. After the fourth test trial, infants immediately moved to the next Training $\rightarrow$ Testing block. The training and testing phases continued until the infant became fussy or until the infant completed six blocks.

A random sample of $25 \%$ of the infants was coded offline. Offline coders watched video streams of the infants and recorded infants' visual fixations at a resolution of 30 frames per second. Reliabilities for online and offline coders were calculated for each infant and averaged across the three conditions, average $r=.90$.

\section{Results and Discussion}

The whole procedure consisted of 12 familiarization trials (96 s) and 24 testing trials (192 s) with varying amounts of time between trials (contingent on a look to the fixation stimulus), and many infants did not make it through all six blocks. The reported ANOVA only includes those infants who completed all six blocks, however, we also report data for all infants who completed at least two training $\rightarrow$ testing blocks (four training trials and eight testing trials). Nine infants in the silent condition, nine infants in the label condition, and eight infants in the sound condition completed all six blocks. The average number of blocks completed did not differ across condition, $F(2,75)=0.68, p=.509, \mathrm{\eta p}^{2}=.02$, with infants in the silent condition completing 4.60 blocks, infants in the label condition completing 4.58 blocks, and infants in the sound condition completing 4.49 blocks.

On each training block infants were presented with two referents, each presented one at a time for $8 \mathrm{~s}$. Thus, within each training block infants could accumulate up to $16 \mathrm{~s}$ of looking. A 2 (Age: 14-months, 22-months) x 3 (Stimulus condition: Silent, Word, and Sound) x 6 (Trial Block: Blocks $1-6$ ) mixed-factors ANOVA with Trial Block manipulated within subjects revealed a significant effect of Stimulus Condition, $F(2,20)=9.03, p=.002, \eta_{p}{ }^{2}$ $=.47$. Infants in the sound condition $(M=14.26 s, S E=0.72 s)$ and word condition $(M=$ $14.25 s, S E=0.68 s$ ) accumulated more looking during training than infants in the silent condition $(M=10.69 s, S E=0.68 s)$, pairwise comparisons with Bonferroni adjustment, $p$ s $<.005, d s>1.49$. The above analysis also revealed a significant effect of Training Block, $F$ $(5,100)=6.33, p<.001, \eta_{\mathrm{p}}^{2}=.24$, and a Training Block x Stimulus Condition interaction, $F(10,100)=2.99, p=.002, \eta_{\mathrm{p}}^{2}=.23$. Infants in Silent condition looked significantly less on the last three training blocks $\left(M_{\text {Blocks } 4-6}=8.76 s, S E=0.96 s\right)$ than on the first three training blocks $\left(M_{\text {Blocks } 1-3}=12.81 s, S E=0.74 s\right), t(8)=4.89, p<.001, d=1.58$. Infants in the word and sound conditions did not significantly decrease looking across training, $p \mathrm{~s}>$. 12. The main effect of age and interactions with age did not approach significance, $p$ 's $>.36$.

\section{Testing Phase Analyses}

On each $8 \mathrm{~s}$ test trial, infants were presented with two stimuli. One stimulus was identical to training and one stimulus was always novel (location varied from trial to trial). We calculated a novelty preference score on each trial (accumulated looking to novel image/ 
accumulated looking to both images), and calculated a mean novelty percent score for each testing block (averaged across the four test trials). Percent looking to the novel images broken up by testing block and stimulus condition are presented in Figure 7a (all infants) and Figure $7 \mathrm{~b}$ (only those infants who completed all six blocks). As can be seen in the Figure $7 \mathrm{a}$, infants in the silent and word conditions were reliably looking to the novel images across all six testing blocks, Blocks $1-6$ greater than 50\%, one-sample $t \mathrm{~s}>2.17, p \mathrm{~s}<.041$. In contrast, infants in the sound condition did not reliably show a novelty preference until Block 3, Blocks $3-6$ greater than $50 \%$, one-sample $t s>2.57, p s<.037$. Thus, infants in the sound condition required more familiarization before showing a novelty preference. As with training data, we also submitted their mean novelty preferences to a 2 (Age: 14-months, 22months) x 3 (Stimulus condition: Silent, Word, and Sound) x 6 (Trial Block: Blocks 1 -6) mixed-factors ANOVA with Trial Block manipulated within subjects. However, due to small sample sizes, none of the effects reached significance.

Additional analyses focused on individual infants' responses across the different stimulus conditions. The following analyses focused on the percentage of infants that demonstrated any reliable novelty (or familiarity) preference, as indicated by the presence of a critical run (see Robinson \& Sloutsky, 2007a; Roder et al., 2000, for similar procedures). While group analyses provide some evidence of different learning rates across the conditions, critical run analysis also provide insight into individual learning rates and they can also help clarify chance performance. For example, as a group, infants could initially be a chance because many of the infants were not discriminating the trained items from new items (consistent with auditory dominance) or they could be at chance because some infants showed a novelty preference, whereas, other infants showed a familiarity preference. The critical run analysis can address this issue because it does not differentiate between reliable novelty and familiarity responders, both are discriminators.

A novelty preference score was averaged across blocks of three consecutive trials (e.g., Block $1=$ Trials $1-3$, Block $2=$ Trials $2-4$, etc.), and a critical run was identified as five consecutive blocks where looking to the novel (or familiar) stimuli exceeded $60 \%$. To determine if words and sounds affected the number of infants who contributed a critical run, we used a chi square to directly compare the two auditory conditions to the unimodal baseline. Only $37 \%$ of the infants in the sound condition contributed a critical run, whereas, $60 \%$ of the infants in the silent baseline contributed a critical run, $\chi^{2}(1, N=52)=2.74, p$ $=.097$. Infants in the label condition were marginally below the baseline, $\chi^{2}(1, N=51)=$ $3.30, p=.069$, with only $35 \%$ of the infants in the label condition contributing a critical run. While the direction of these effects point to overshadowing, they did not reach significance when using an alpha level of .05.

In summary, infants in the sound and word conditions accumulated more looking to the images than infants in the silent condition, however, this increased looking did not facilitate or speed up visual processing. Rather, individual and group analyses both provide some evidence that unfamiliar sounds may have disrupted the encoding of visual images, as indicated by infants requiring more familiarization before showing a reliable novelty (or familiarity) preference. Given that the auditory stimulus was not presented during the testing phase, these effects are best accounted for by auditory input overshadowing visual input. 
Effects of words on visual processing speed were less robust. While group analyses showed no differences between infants in the word and silent conditions, individual analyses provide some support that a higher proportion of infants in the silent condition showed a reliable novelty preference.

\section{General Discussion}

By manipulating whether auditory input was present or absent during the response/decision phase, the current study provides important insights into the mechanisms underlying auditory dominance effects. Recall that we trained children in Experiments $1 \mathrm{~A}$ and $2 \mathrm{~A}$ on auditory-visual pairings and then tested recognition of the visual stimuli that were accompanied by new or old auditory stimuli. When new visual images were paired with old auditory stimuli, children in both experiments were less likely to notice the new images compared to children who saw the same images presented in isolation. This suggests that the auditory input either disrupted the encoding of the visual stimuli (overshadowing) or interfered with visual processing during the decision/response phase (response competition). In Experiments 1B and 2B, children were trained on the same auditory-visual pairings, however, visual images were presented in silence when they were required to make a response. Under these manipulations, children ably discriminated the visual stimuli, and visual discrimination was comparable in the cross-modal and unimodal conditions. These findings suggest that children do encode the visual images and that auditory dominance effects reported in Experiments 1A and 2A stem from auditory input interfering with their decision or response, rather than from auditory input disrupting the encoding of visual images.

Experiment 3 presented 14- and 22-month-olds with auditory-visual pairings and then tested recognition of the visual images at various points in the course of processing. While removing the auditory stimulus from the testing phase attenuated auditory dominance effects in young children, 14- and 22-month-olds still had difficulty processing the visual input under these testing conditions. In particular, infants who saw visual images paired with unfamiliar sounds were slower at processing the visual images compared to infants who saw the same images presented in silence. At the group level, there was no slowdown for infants who heard labels during training. Examination of individual responses may suggest that words presented in a word learning context may have also disrupted visual processing. In particular, while $60 \%$ of infants in the silent condition reliably discriminated the referents from novel stimuli, only $35 \%$ of the infants made this discrimination in the label condition. However, this effect was only marginally significant, thus, future research will need to corroborate these potential interference effects with a larger sample.

The current findings have implications on research examining the development of crossmodal processing, and modality dominance effects more specifically, and are likely to have implications on many higher-order tasks that hinge on cross-modal processing.

\section{Cross-modal processing and Auditory Dominance effects}

Infants and young children live in a multimodal world where they constantly encounter information presented to multiple sensory modalities. Under some situations, presenting 
cross-modal information can facilitate learning (Bahrick, Flom, \& Lickliter, 2002; Bahrick \& Lickliter, 2000), whereas under other situations, cross-modal presentation can sometimes lead to modality dominance, with one modality dominating processing in another modality. Many mechanisms have been introduced to account for modality dominance effects, however, most of these mechanisms have been put forward to account for visual dominance effects in adults when using variations of the Colavita visual dominance task (Colavita, 1974; Colavita et al., 1976; Colavita \& Weisberg, 1979; Egeth \& Sager, 1978; Koppen et al., 2008; Ngo et al., 2010; Ngo et al., 2011; Sinnett et al., 2008; Sinnett et al., 2007; see also Spence, 2009; Spence et al., 2012, for reviews). Consistent with developmental research examining modality dominance (Nava \& Pavani, 2013; Robinson \& Sloutsky; 2004; Sloutsky \& Napolitano, 2003, see also Hirst et al., 2018; Robinson \& Sloutsky, 2010a, for reviews), the current study found support for auditory dominance in young children with no evidence that cross-modal presentation had a greater cost on auditory processing. Thus, it is doubtful that any of the proposed mechanisms underlying visual dominance can account for the current findings.

The primary goal of the current study was to determine what mechanism(s) best accounts for auditory dominance in infants and young children, and effects of auditory input on visual processing more generally. Recall that we introduced two mechanisms that could potentially account for attenuated visual processing: Overshadowing and response competition.

According to the auditory overshadowing account, children have difficulty attending to simultaneously presented auditory and visual information. Furthermore, it is often argued that early in development auditory input is more likely to engage attention and attenuate or delay the encoding of a corresponding visual stimulus (Sloutsky \& Napolitano, 2003; Robinson \& Sloutsky, 2004, see also Robinson \& Sloutsky, 2007a; 2010a; Sloutsky \& Robinson, 2008, for related discussions). Such an account assumes that the modality that is faster to engage attention (most often auditory) dominates the other modality early in the course of processing.

According to the response competition account, children do not have difficulty encoding cross-modal stimuli. Rather, attenuated visual processing may occur relatively late in the course of processing with children primarily relying on the auditory stimulus when making comparisons between two cross-modal stimuli. While details of this account are not known, we will briefly speculate on the underlying mechanism. In Experiment 1 children had to either compare two cross-modal stimuli in memory or compare a cross-modal stimulus that was currently being presented with one stored in memory. It is possible that making comparisons between two auditory stimuli and two visual stimuli exceeds young children's working memory capacity and therefore comparisons are computed serially. According to this account, children in cross-modal conditions may compare auditory stimuli and make a response before comparisons are made in the visual modality. Thus, removing the auditory stimulus at test may have given children the opportunity to compare the visual stimuli before making a response.

At least two additional accounts need to be eliminated before any strong conclusions can be made regarding children's serial retrieval of auditory and visual information from memory. First, it is possible that stimulus complexity may also underlie auditory dominance, with 
auditory stimuli being easier to encode than complex visual stimuli. While the current study cannot eliminate this account, previous research has systematically manipulated familiarity and complexity, and familiarity was a better predictor of modality dominance (e.g., Napolitano \& Sloutsky, 2004). Thus, it is possible that when cross-modal stimuli differ in relative familiarity, familiar items are retrieved faster or before unfamiliar items.

Alternatively, it is also possible that auditory stimuli may dominate because they are still in sensory memory, whereas, visual stimuli are likely to decay at a faster rate (Darwin, Turvey, \& Crowder, 1972; Sperling, 1960). Thus, it is possible that when children in Experiment 1 were determining if two auditory-visual pairs were the same or different, they may rely more on the auditory stimulus because it is still in sensory memory, whereas, much of the details of the visual stimulus had already decayed. However, this account would have difficulty explaining findings of Experiments 2 and 3 where both auditory and visual information should have had time to decay from sensory memory.

The current study may also suggest that overshadowing and response competition may have different developmental trajectories, with overshadowing driving effects in young infants (Robinson \& Sloutsky, 2004; 2007a; 2010b; Sloutsky \& Robinson, 2008) and response competition driving many of the effects in older children (Napolitano \& Sloutsky, 2004; Sloutsky \& Napolitano, 2003). While such a conclusion is warranted given the current findings, future research will need to systematically tease these mechanisms apart before any strong conclusions can be drawn. For example, using more sensitive measures such as latency of visual P300's in passive oddball tasks (Robinson, Ahmar, \& Sloutsky, 2010) and response times and latencies of first fixations in change detection tasks such as the one reported in Experiment 1A (Barnhart, Rivera, \& Robinson, 2018; Dunifon, Rivera, \& Robinson, 2016; Robinson, Chandra, \& Sinnett, 2016), recent findings provide some support for auditory dominance in children and young adults. For example, while pairing auditory and visual stimuli together slowed down visual P300, response times detecting changes in visual information, and the latency of first fixations to the visual item, it often had no effect or sped up auditory processing. Thus, while auditory overshadowing effects appear to weaken with age, these effects are still present under certain testing conditions.

Distinguishing between overshadowing and response competition may also account for the finding that stimulus familiarity appears to have a different effect at various points in development. In particular, increasing the familiarity of the auditory stimulus often results in stronger auditory dominance effects in young children (Napolitano \& Sloutsky, 2004), whereas, increasing the familiarity of the auditory stimulus often attenuates auditory dominance effects in young infants (Robinson \& Sloutsky, 2007a; 2010b; Sloutsky \& Robinson, 2008). One possible explanation that may resolve this discrepancy is by positing that stimulus familiarity has different effects at different points in the course of processing. During encoding, unfamiliar stimuli are likely to consume more attentional resources than familiar stimuli. Assuming that attentional resources are finite, unfamiliar auditory stimuli should be more likely to interfere with the encoding of a visual stimulus than familiar auditory stimuli. However, when the task is to compare a stimulus with another stimulus stored in memory, familiar stimuli may be retrieved faster and more efficiently than unfamiliar stimuli. Furthermore, if comparisons of cross-modal stimuli are computed 
serially in memory, then children may rely on familiar stimuli more than unfamiliar stimuli because it is retrieved faster and/or more efficiently retrieved than unfamiliar stimuli.

\section{Effects of words on higher-order tasks}

While the current study focused primarily on the processing of auditory and visual stimuli, the current findings are likely to have implications on many tasks that hinge on cross-modal processing. For example, in word learning situations, the young word learner must attend to simultaneously presented words and pictures and bind this information. According to parental report, young infants have already started forming some word-object associations as early as 8-months of age (Fenson et al., 1994). Furthermore, it is well established that slightly older infants can be taught arbitrary auditory-visual pairings in the laboratory (Schafer \& Plunkett, 1998). How is it possible to reconcile the current findings with research demonstrating that young infants can quickly learn arbitrary auditory-visual pairings?

One apparent difference between the current study with studies examining early word learning is that the current study tested recognition of the referents, whereas, binding of the auditory and visual stimuli is tested in word learning studies. While some encoding of the referent is needed for binding to occur, full processing of the image may not be necessary. By comparing visual processing in a word learning context to a silent condition in young infants, there are situations when words attenuate visual processing (Robinson \& Sloutsky, 2007a; 2008; Sloutsky \& Robinson, 2008, see also Experiment 3 of the current study). Given these findings, it seems plausible to assume that infants might be mapping words onto partially encoded referents. However, such a claim will require further testing.

While attenuated processing of a referent could be considered as a cost of word learning, it is possible that these effects are fundamental in other contexts. For example, attenuated visual processing could explain how infants embedded in a perceptually-rich environment quickly disregard the fine details of a referent in favor of a more abstract, generic representation (e.g., overall shape, whole object, etc.). From this perspective, labeling objects may not only affect word and category learning through a honing-in process (i.e., increasing attention to specific features relevant to a category) but also through a pruning process in which many of the fine details of a stimulus are automatically removed, thus, leaving the infant with a generic representation of the referent. This perceptually impoverished representation may not only promote generalization and transfer (cf., Kaminski \& Sloutsky, 2013; Kaminski, Sloutsky, \& Heckler, 2009; Smith, 2003; Uttal, Liu, \& DeLoache, 1999), but it may also decrease the discriminability of labeled objects, thus, increasing the likelihood of grouping these objects together (e.g., Sloutsky, Robinson, \& Timbrook, 2005).

Overshadowing and response competition may also account for some of the effects of words on induction tasks. For example, in induction tasks (e.g., Gelman \& Markman, 1986; Sloutsky \& Fisher, 2004), an experimenter labels a target picture and then labels two test pictures. Children's task is to generalize a non-obvious property, and they can rely on appearance (visual input) or on the label (auditory input) to make their generalizations. It is often found that young children are more likely to rely on the label when making generalizations than on appearance. Several different explanations can account for this 
effect. The first possibility is that children understand the conceptual importance of labels, with labels serving as proxies to category membership (Gelman \& Coley, 1991). A second possibility is that auditory input (including words) overshadows or attenuates the encoding of visual input (Sloutsky \& Napolitano, 2003). Finally, the current study demonstrates that auditory stimuli can also interfere during the decision or response phase. While overshadowing and response competition do not eliminate language-specific accounts, these mechanisms offer alternative explanations that ground children's behaviors in the dynamics of cross-modal processing.

\section{Limitations and Future Directions}

While the current findings shed light on potential mechanisms underlying cross-modal processing and auditory dominance, there are also some limitations to the current study which need to be addressed in future research. First, stimuli and methodologies varied across all three reported experiments. Finding the same pattern of results while using a variety of stimuli and testing procedures shows that the findings are generalizable, however, they also make it difficult to fully capture development change. For example, it is unclear if differences between infants and children stem from development/maturation or from methodological differences. Second, there are many factors that could potentially affect encoding, storage, and binding of cross-modal information. For example, the current study found the same pattern of results in Experiments 1 and 2 even though we increased the time between training and test. Increasing the stimulus duration from $1 \mathrm{~s}$ in Experiment 1 to 1.4 to $1.8 \mathrm{~s}$ in Experiment 2 also appeared to have little effect, however, more methodological control is needed before any strong conclusions can be made regarding effects of stimulus duration and inter-stimulus intervals. Third, to the best of our knowledge, there are no other studies that have used a variation of a continuous familiarization procedure to assess how a word learning context affects the speed of encoding of the referents. We believe it is important to better understand this process, as it is possible that low-level dynamics of crossmodal processing may account for a variety of higher-order effects such as effects of linguistic input on cognitive tasks. However, it is clear from the attrition rate in Experiment 3 , that methodological changes are needed to keep infants more engaged throughout the entire procedure. Finally, future research will need to further examine what is driving auditory to visual dominance shifts across development (e.g., Nava \& Pavani, 2013) and changes in overshadowing and response condition. Many factors such as development of reading, better attentional control, increased working memory capacity, and faster processing speed may all affect how cross-modal stimuli are encoded and stored in memory.

\section{Conclusion}

In summary, there are many tasks that require infants and children to process multimodal information. While this ability is often taken for granted, there are many occasions where auditory input disrupts visual processing. The current study demonstrates that sounds, words presented in isolation, and words embedded in a word learning scenario can all attenuate visual processing. These effects can stem from auditory input attenuating the encoding of visual input (auditory overshadowing) and from auditory input interfering with visual processing during the decision/response phase (response competition). Examining these mechanisms in future research will shed light on how attention is allocated to cross-modal 
stimuli and will possibly ground many sophisticated behaviors in low-level processes of cross-modal processing.

\section{Acknowledgments}

This research is supported by NIH grants R01HD078545 and P01HD080679 to VMS and by NIH grant R03HD055527 to CWR.

\section{References}

Althaus N, \& Plunkett K (2015). Categorization in infancy: Labeling induces a persisting focus on commonalities. Developmental Science, 19, 770-780. [PubMed: 26538010]

Althaus N, \& Westermann G (2016). Labels constructively shape object categories in 10-month-old infants. Journal of Experimental Child Psychology, 151, 5-17. [PubMed: 26778468]

Bahrick LE, \& Lickliter R (2000). Intersensory redundancy guides attentional selectivity and perceptual learning in infancy. Developmental Psychology, 36(2), 190-201. [PubMed: 10749076]

Bahrick LE, Flom R, \& Lickliter R (2002). Intersensory redundancy facilitates discrimination of tempo in 3-month-old infants. Developmental Psychology, 41(4), 352-363.

Balaban MT, \& Waxman SR (1997). Do words facilitate object categorization in 9-monthold infants? Journal of Experimental Child Psychology, 64, 3-26. [PubMed: 9126625]

Baldwin DA, \& Markman EM (1989). Establishing word-object relations: A first step. Child Development, 60, 381-398. [PubMed: 2924658]

Barnhart WR, \& Rivera S, \& Robinson CW (2018). Different patterns of modality dominance across development. Acta Psychologica, 182, 154-165. [PubMed: 29179020]

Birnholz JC, \& Benaceraff BB (1983). The development of human fetal hearing. Science, 222, 516518. [PubMed: 6623091]

Colavita FB (1974). Human sensory dominance. Perception \& Psychophysics, 16, 409-412.

Colavita FB, Tomko R, \& Weisberg D (1976). Visual pre-potency and eye orientation. Bulletin of the Psychonomic Society, 8, 25-26.

Colavita FB, \& Weisberg D (1979). A further investigation of visual dominance. Attention, Perception \& Psychophysics, 25, 345-347. [PubMed: 461094]

Colonius H, \& Diederich A (2006). The race model inequality: Interpreting a geometric measure of the amount of violation. Psychological Review, 113(1), 148-154. [PubMed: 16478305]

Darwin CJ, Turvey MT, \& Crowder RG (1972). An auditory analogue of the Sperling partial report procedure: Evidence for brief auditory storage. Cognitive Psychology, 3, 255-267.

Desimone R, \& Duncan J (1995). Neural mechanisms of selective visual attention. Annual Review of Neuroscience, 18, 193-222.

Duncan J (1996). Cooperating brain systems in selective perception and action In Inui T \& McClelland JL (Eds.), Attention and performance XVI: Information integration in perception and communication (pp. 549-578). Cambridge, MA: The MIT Press.

Duncan J, Martens S, \& Ward R (1997). Restricted attentional capacity within but not between sensory modalities. Nature, 387, 808-810. [PubMed: 9194561]

Dunifon C, Rivera S, \& Robinson CW (2016). Auditory stimuli automatically grab attention: Evidence from eye tracking and attentional manipulations. Journal of Experimental Psychology: Human Perception and Performance, 42, 1947-1958. [PubMed: 27505224]

Egeth HE, \& Sager LC (1977). On the locus of visual dominance. Attention, Perception \& Psychophysics, 22, 77-86.

Eimer M, \& Driver J (2000). An event-related brain potential study of cross-modal links in spatial attention between vision and touch. Psychophysiology, 37(05), 697-705. [PubMed: 11037046]

Eimer M, \& van Velzen J (2002). Cross-modal links in spatial attention are mediated by supramodal control processes: Evidence from event- related brain potentials. Psychophysiology, 39, 437-449. [PubMed: 12212636] 
Fantz RL (1964). Visual experience in infants: Decreased attention to familiar patterns relative to novel ones. Science, 46, 668-670.

Fenson L, Dale PS, Reznik JS, Bates E, Thal E, \& Plethick JP (1994). The MacAurthur Communicative Development Inventories. Monographs of the Society for Research in Child Development, Serial no. 242, vol. 59(5).

Ferry AL, Hespos SJ, \& Waxman SR (2010). Categorization in 3- and 4-month-old infants: an advantage of words over tones. Child Development, 81, 472-479. [PubMed: 20438453]

Fort A, Delpuech C, Pernier J, \& Giard MH (2002). Dynamics of cortico-subcortical cross-modal operations involved in audio-visual object recognition in humans. Cerebral Cortex, 12, 10311039. [PubMed: 12217966]

Fulkerson AL, \& Haaf RA (2003). The influence of labels, non-labeling sounds, and source of auditory input on 9- and 15-month-olds' object categorization. Infancy, 4, 349-369.

Gelman SA, \& Coley J (1991). Language and categorization: The acquisition of natural kind terms In Gelman SA, \& Byrnes JP (Eds.). Perspectives on language and thought: Interrelations in development (146-196). New York: Cambridge University Press.

Gelman SA, \& Markman E (1986). Categories and induction in young children. Cognition, 23, 183209. [PubMed: 3791915]

Giard MH, \& Peronnet F (1999). Auditory-visual integration during multimodal object recognition in humans: A behavioral and electrophysiological study. Journal of Cognitive Neuroscience, 11, 473490. [PubMed: 10511637]

Hirst R, Cragg L, \& Allen HA (2018, 3 27). Vision dominates audition in adults but not children: A meta-analysis of the Colavita effect. http://doi.org/10.17605/OSF.IO/M42N3

Jusczyk PW (1998). The discovery of spoken language. Cambridge, MA: MIT Press.

Kaminski JA, \& Sloutsky VM (2013) Extraneous perceptual information interferes with children's acquisition of mathematical knowledge. Journal of Educational Psychology, 105, 351-363.

Kaminski JA, Sloutsky VM, \& Heckler AF (2009) Transfer of mathematical knowledge: The portability of generic instantiations. Child Development Perspectives, 3, 151-155.

Koppen C, Alsius A, \& Spence C (2008). Semantic congruency and the Colavita visual dominance effect. Experimental Brain Research, 184, 533-546. [PubMed: 17885751]

Koppen C, \& Spence C (2007). Audiovisual asynchrony modulates the Colavita visual dominance effect. Brain Research, 1186, 224-232. [PubMed: 18005944]

Lewkowicz DJ (1988a). Sensory dominance in infants: 1. Six-month-old infants' response to auditoryvisual compounds. Developmental Psychology, 24, 155-171.

Lewkowicz DJ (1988b). Sensory dominance in infants: 2. Ten-month-old infants' response to auditoryvisual compounds. Developmental Psychology, 24, 172-182.

Massaro DW (1984). Children's perception of visual and auditory speech. Child Development, 55, 1777-1788. doi:10.2307/1129925 [PubMed: 6510054]

Miller J (1982). Divided attention: Evidence for coactivation with redundant signals. Cognitive Psychology, 14, 247-279. [PubMed: 7083803]

Mishra J, Martinez A, \& Hillyard SA (2008). Cortical processes underlying sound-induced flash fusion. Brain Research, 1242, 102-115. [PubMed: 18585695]

Mishra J, Martinez A, Sejnowski TJ, and Hillyard SA (2007). Early cross-modal interactions in auditory and visual cortex underlie a sound-induced visual illusion. Journal of Neuroscience, 27, 4120-4131. [PubMed: 17428990]

Napolitano AC, \& Sloutsky VM (2004). Is a picture worth a thousand words? The flexible nature of modality dominance in young children. Child Development, 75, 1850-1870. [PubMed: 15566384]

Nava E, \& Pavani F (2013). Changes in sensory dominance during childhood: Converging Evidence from the Colavita Effect and the Sound-Induced Flash Illusion. Child Development, 84(2), 604616. [PubMed: 23006060]

Ngo MK, Cadieux ML, Sinnett S, \& Soto-Faraco S (2011). Reversing the Colavita visual dominance effect. Experimental Brain Research, 214(4), 607-618. [PubMed: 21909983]

Ngo MK, Sinnett S, Soto-Faraco S, \& Spence C (2010). Repetition blindness and the Colavita effect. Neuroscience Letters, 480, 186-190. [PubMed: 20561565] 
Parker JL, \& Robinson CW (2018). Changes in multisensory integration across the lifespan. Psychology and Aging, 33(3) 545-558. [PubMed: 29756807]

Pavani F, Husain M, Ládavas E, \& Driver J (2004). Auditory deficits in visuospatial neglect patients. Cortex, 40(2), 347-365. [PubMed: 15156793]

Plunkett K, F., H. J., \& Cohen LB (2008). Labels can override perceptual categories in early infancy. Cognition, 106, 665-681. [PubMed: 17512515]

Posner MI, Nissen MJ, \& Klein RM (1976). Visual dominance: An information-processing account of its origins and significance. Psychological Review, 83, 157-171. [PubMed: 769017]

Robinson CW, Ahmar N, \& Sloutsky VM (2010). Evidence for auditory dominance in a passive oddball task. In Ohlsson S \& Catrambone R (Eds.), Proceedings of the 32nd Annual Conference of the Cognitive Science Society (pp 2644-2649). Austin, TX: Cognitive Science Society.

Robinson CW, Best CA, Deng S, \& Sloutsky VM (2012). The Role of Words in Cognitive Tasks: What, When, and How? Frontiers in Psychology, 3, 1-8. [PubMed: 22279440]

Robinson CW, Chandra M, \& Sinnett S (2016). Existence of competing modality dominances. Attention, Perception, \& Psychophysics, 78, 1104-1114.

Robinson CW, \& Sloutsky VM (2004). Auditory dominance and its change in the course of development. Child Development, 75, 1387-1401. [PubMed: 15369521]

Robinson CW, \& Sloutsky VM (2007a). Visual processing speed: Effects of auditory input on visual processing. Developmental Science, 10, 734-740. doi: 10.1111/j.1532-7078.2007.tb00225.x [PubMed: 17973789]

Robinson CW, \& Sloutsky VM (2007b). Linguistic labels and categorization in infancy: Do labels facilitate or hinder? Infancy, 11, 233-253. doi: 10.1111/j.1532-7078.2007.tb00225.x

Robinson CW, \& Sloutsky VM (2008). Effects of auditory input in individuation tasks. Developmental Science, 11, 869-881. [PubMed: 19046156]

Robinson CW, \& Sloutsky VM (2010a). Development of cross-modal processing. Wiley Interdisciplinary Reviews: Cognitive Science, 1(1), 135-141. [PubMed: 26272846]

Robinson CW \& Sloutsky VM (2010b). Effects of multimodal presentation and stimulus familiarity on auditory and visual processing. Journal of Experimental Child Psychology, 107, 351-358. [PubMed: 20553691]

Robinson CW, \& Sloutsky VM (2013). When audition dominates vision: Evidence from cross-modal statistical learning. Experimental Psychology, 60(2), 113-121. [PubMed: 23047918]

Roder BJ, Bushnell EW, \& Sasseville AM (2000). Infants' preferences for familiarity and novelty during the course of visual processing. Infancy, 1(4), 491-507.

Rutschmann J, \& Link R (1964). Perception of temporal order of stimuli differing in sense mode and simple reaction time. Perceptual and Motor Skills, 18, 345-352. [PubMed: 14166017]

Schafer G, \& Plunkett K (1998). Rapid word learning by 15 -month-olds under tightly controlled conditions. Child Development, 69, 309-320. [PubMed: 9586207]

Sereno MI, Dale AM, Reppas JB, Kwong KK, Belliveau JW, Brady TJ, Tootell RB (1995). Borders of multiple visual areas in humans revealed by functional magnetic resonance imaging. Science, 268 , 889-893. [PubMed: 7754376]

Shams L, Kamitani Y, \& Shimojo S (2000). Illusions: What you see is what you hear. Nature, 408, 788. doi:10.1038/35048669 [PubMed: 11130706]

Shams L, Kamitani Y, \& Shimojo S (2002). Visual illusion induced by sound. Cognitive Brain Research, 14, 147-152. [PubMed: 12063138]

Sinnett S, Soto-Faraco S, Spence S (2008). The co-occurrence of multisensory competition and facilitation. Acta Psychologica, 128, 153-161. [PubMed: 18207117]

Sinnett S, Spence C, \& Soto-Faraco S (2007). Visual dominance and attention: Revisiting the Colavita effect. Perception \& Psychophysics, 69, 673-686. [PubMed: 17929691]

Sloutsky VM, \& Fisher AV (2004). Induction and categorization in young children: A similarity-based model. Journal of Experimental Psychology: General, 133, 166-188. [PubMed: 15149249]

Sloutsky VM, \& Napolitano A (2003). Is a picture worth a thousand words? Preference for auditory modality in young children. Child Development, 74, 822-833. [PubMed: 12795392] 
Sloutsky VM, \& Robinson CW (2008). The role of words and sounds in visual processing: From overshadowing to attentional tuning. Cognitive Science, 32, 354-377.

Sloutsky VM, Robinson CW, \& Timbrook CM (2005). Overshadowing as a mechanism underlying the effect of labels on categorization. In Bara BG, Barsalou L, \& Bucciarelli M (Eds.), Proceedings of the 27th Annual Conference of the Cognitive Science Society, 2033-2037 Mahwah, NJ: Erlbaum.

Smith LB (2003). Learning to recognize objects. Psychological Science, 14, 244-250. [PubMed: $12741748]$

Snodgrass JG, \& Corwin J (1988). Pragmatics of measuring recognition memory: Applications to dementia and amnesia. Journal of Experimental Psychology: General, 117, 34-50. [PubMed: 2966230]

Spence C (2009). Explaining the Colavita visual dominance effect, Progress in Brain Research, 176, 245-258. [PubMed: 19733761]

Spence C, Parise C, \& Chen YC (2012). The Colavita visual dominance effect In Murray MM, \& Wallace MT (Eds.), The Neural Bases of Multisensory Processes (pp. 529-556). Boca Raton, FL: CRC Press.

Sperling G (1960). The information available in brief visual presentations. Psychological Monographs, 74 (II, Whole No. 498), 1-29.

Uttal DH, Liu LL, \& DeLoache JS (1999). Taking a hard look at concreteness: Do concrete objects help young children learn symbolic relations? In Tamis-LeMonda CS (Ed.), Child psychology: A handbook of contemporary issues (pp. 177-192). Philadelphia, PA: Psychology Press.

Waxman SR (2003). Links between object categorization and naming: Origins and emergence in human infants In Rakison DH, \& Oakes LM (Eds.), Early category and concept development: Making sense of the blooming, buzzing confusion (pp. 213-241). NY, New York: Oxford University Press.

Welch RB \& Warren DH (1980). Immediate perceptual response to Intersensory discrepancy. Psychological Bulletin, 88, 638-667. [PubMed: 7003641]

Wickens CD (1984). Processing resources in attention In Parasuraman R \& Davies R (Eds.), Varieties of attention (pp. 63-101). New York: Academic Press.

$\mathrm{Xu} F$ (2002). The role of language in acquiring object kind concepts in infancy. Cognition, 85, 223250. [PubMed: 12169410] 


\section{Highlights}

- $\quad$ Research highlights situations where auditory input attenuates visual processing/responding.

- In children, auditory interference stems from response competition.

- In young infants, auditory interference may stem from disrupted visual encoding.

- $\quad$ Findings shed light on mechanisms underlying auditory dominance.

- Findings have implications on tasks that hinge on multisensory processing. 


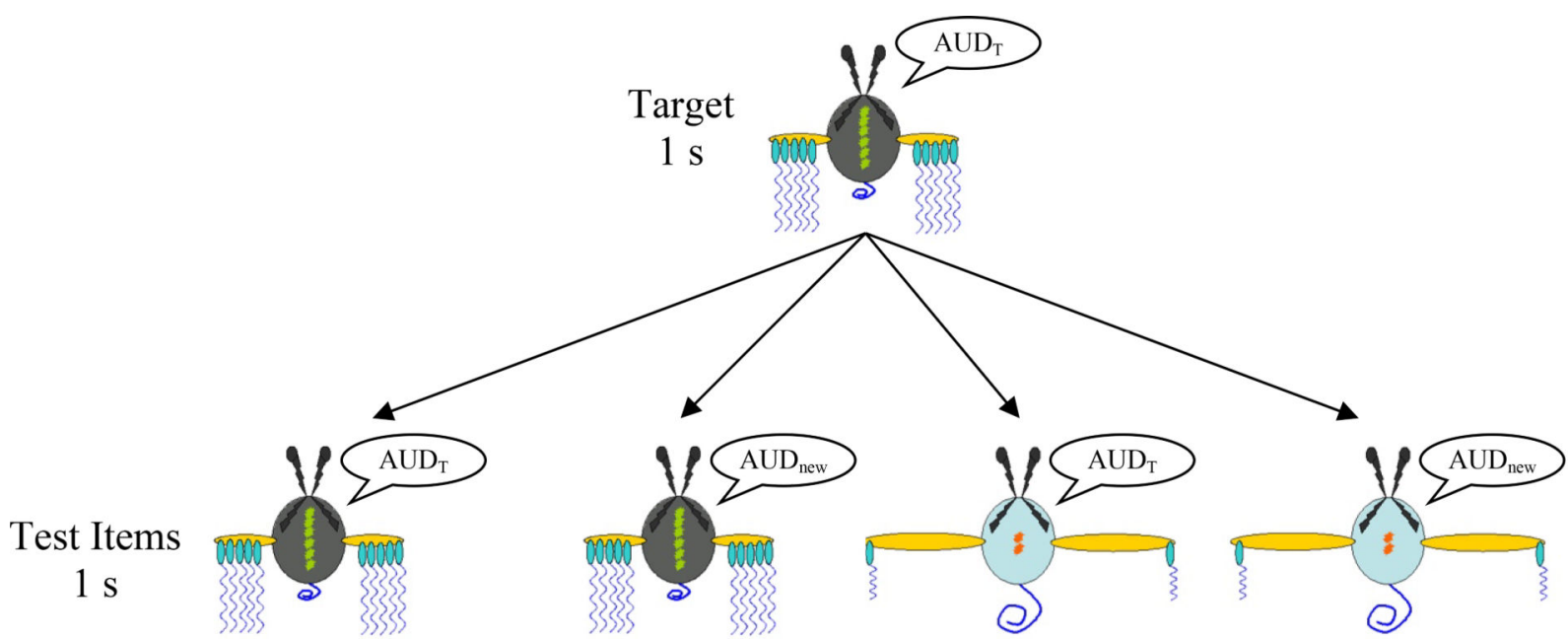

\begin{tabular}{|c|c|c|c|}
\hline Same & $\begin{array}{c}\text { Different Auditory- } \\
\text { Same Visual }\end{array}$ & $\begin{array}{c}\text { Different Visual- } \\
\text { Same Auditory }\end{array}$ & Both Different \\
\hline is "same" & & is "different" & \\
\hline
\end{tabular}

Figure 1.

Examples of visual stimuli and overview of test trial types in Experiment 1A. 


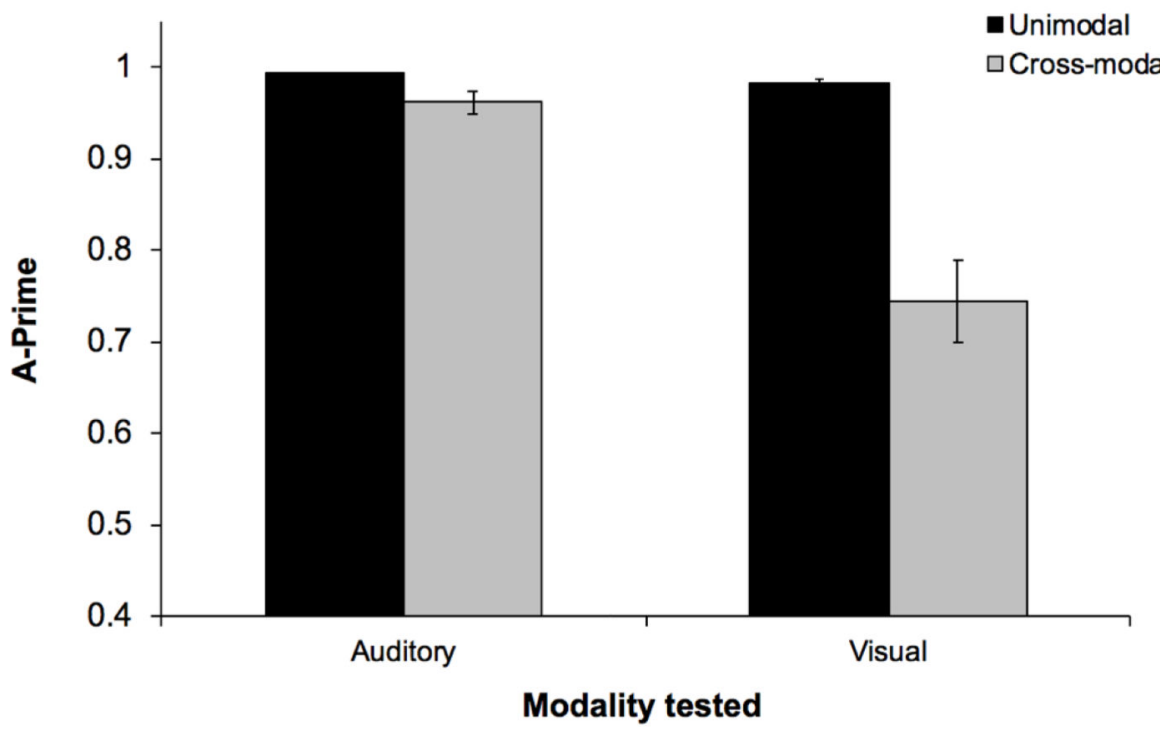

Figure 2.

Discrimination of auditory and visual stimuli in the unimodal and cross-modal conditions in Experiment 1A. Error bars represented Standard Errors. 


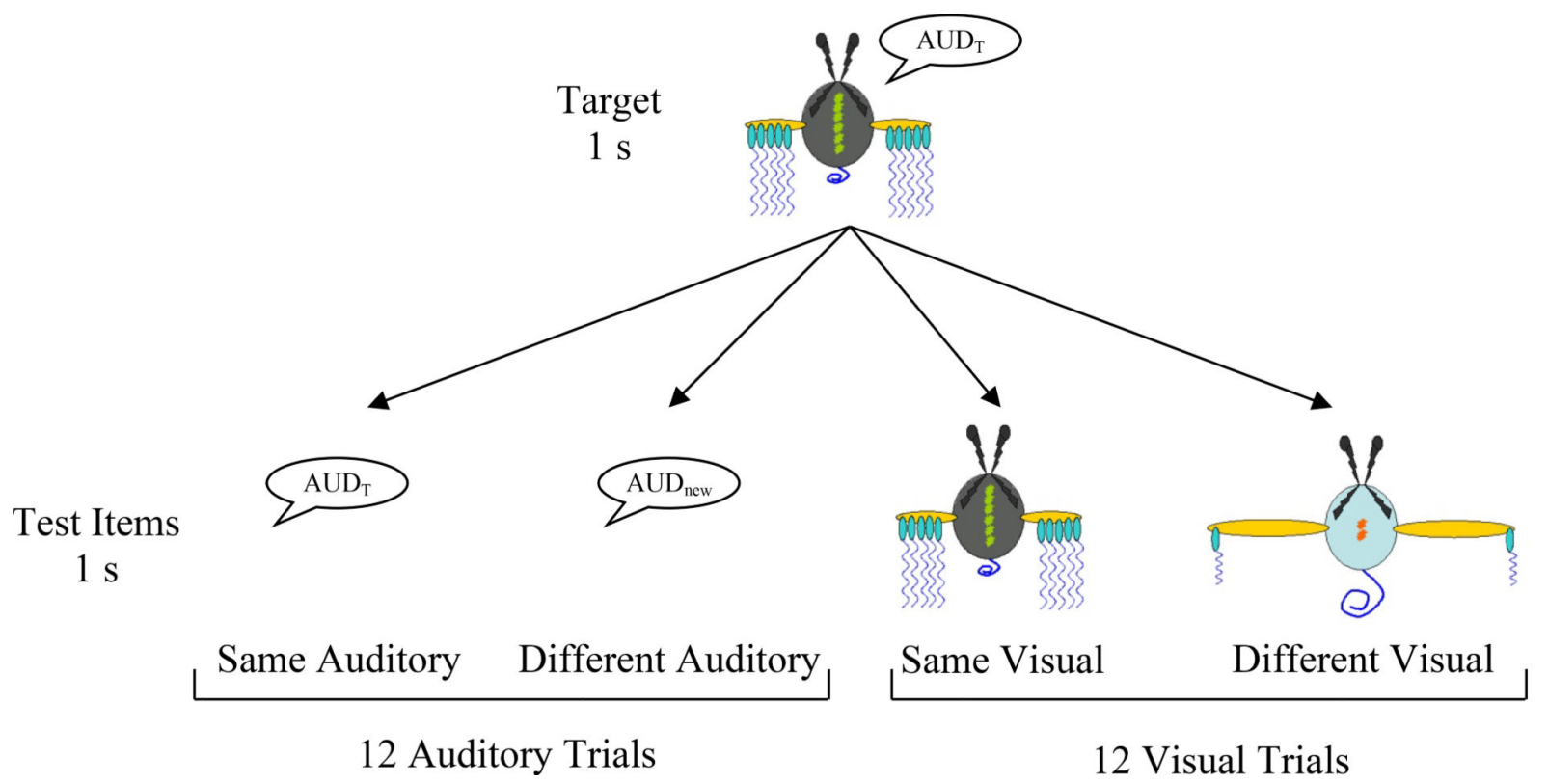

Figure 3.

Examples of visual stimuli and overview of test trial types in Experiment 1B. 


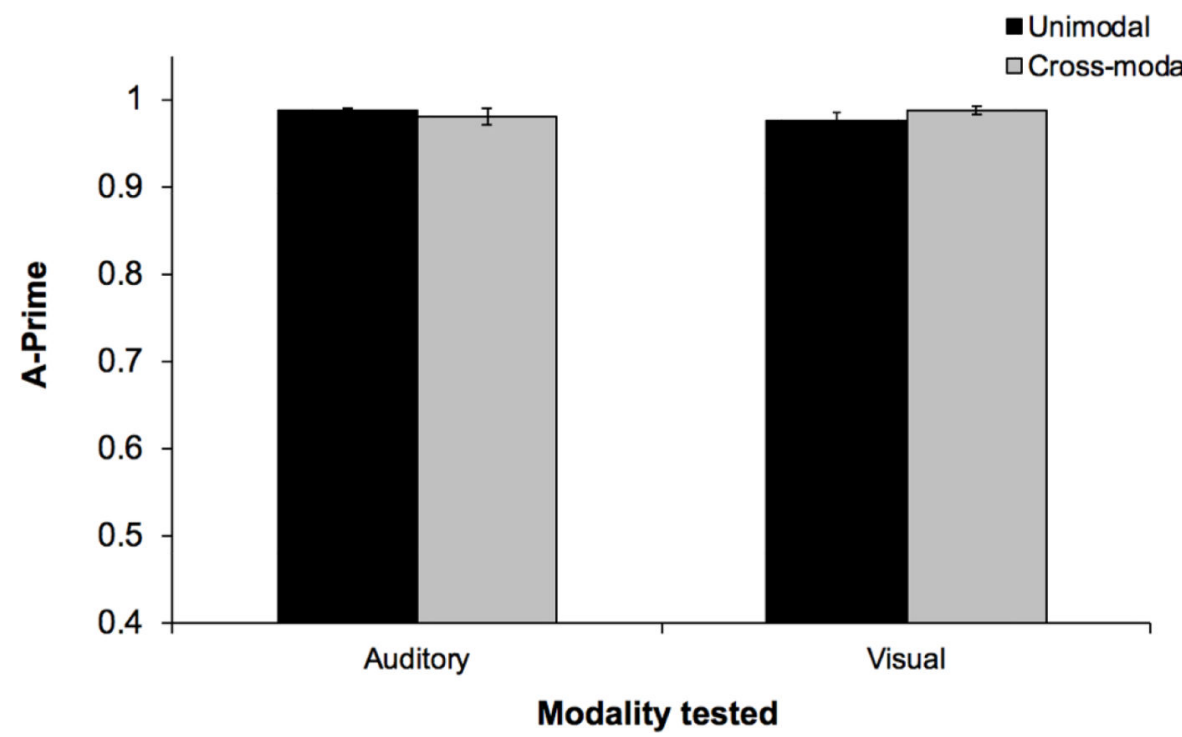

Figure 4.

Discrimination of auditory and visual stimuli in the unimodal and cross-modal conditions in Experiment 1B. Error bars represented Standard Errors. 

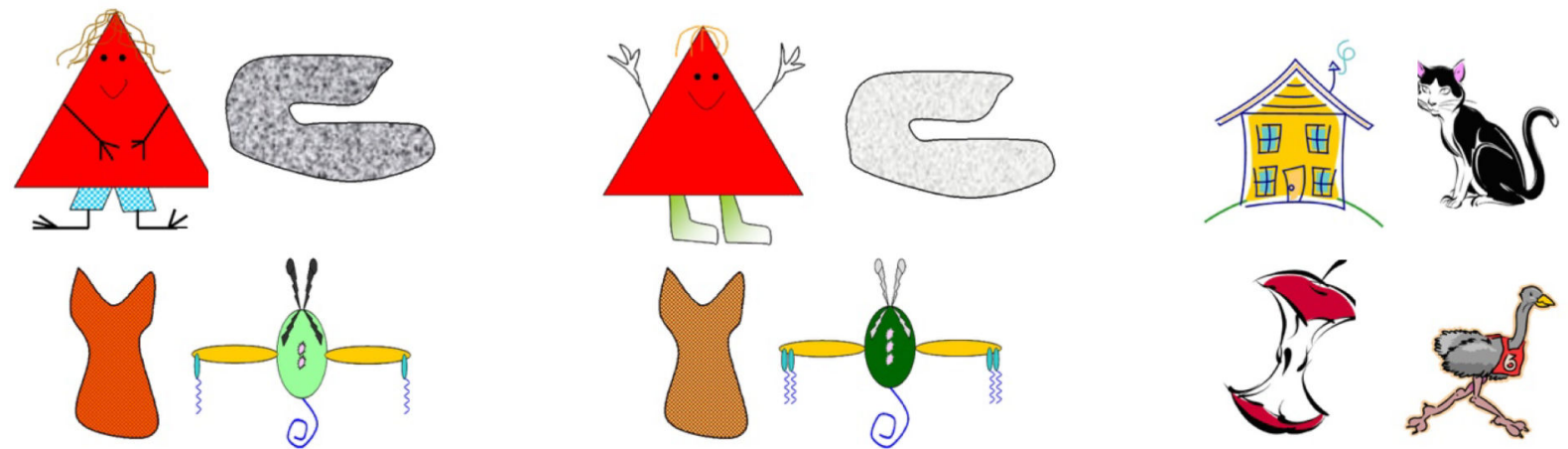

Referents

Examples of Critical lures
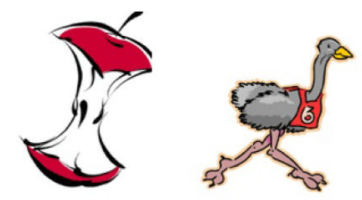

Examples of Catchers

Figure 5.

Examples of visual stimuli presented in Experiments 2A and 2B. 

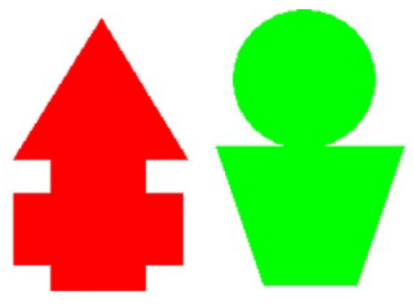

Referents

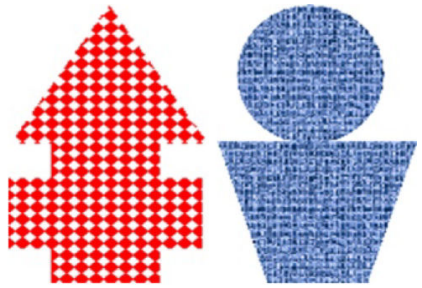

Examples of

Critical lures

Figure 6.

Examples of visual stimuli presented in Experiment 3. 
(a)

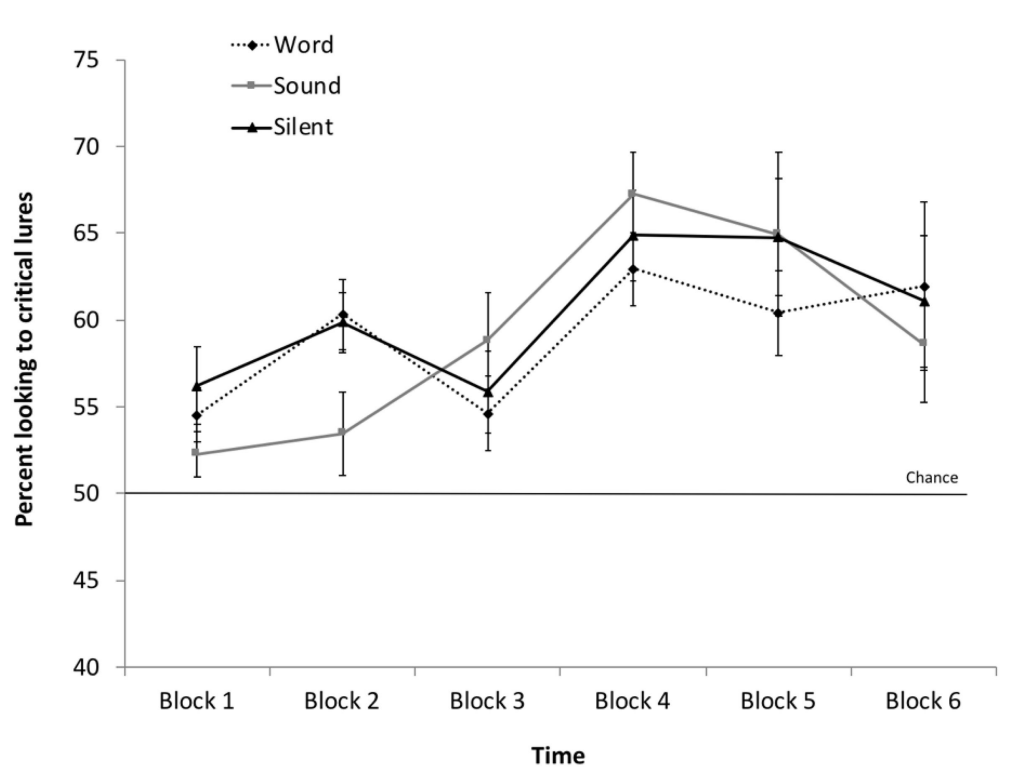

(b)

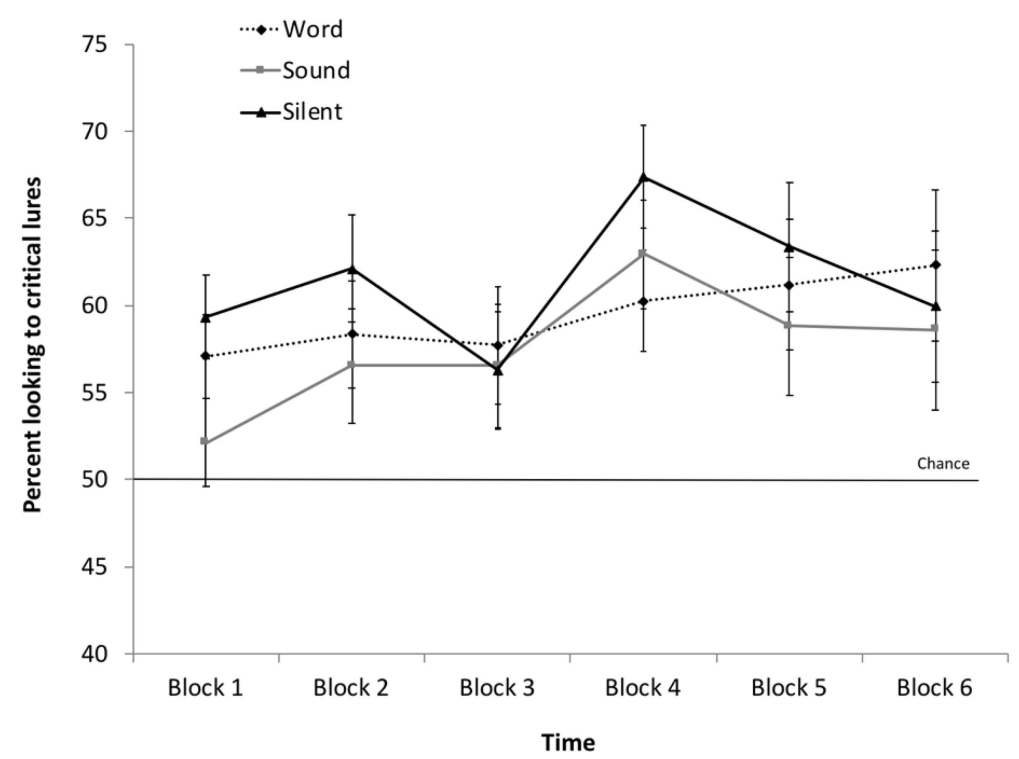

Figure 7.

Percent looking to novel stimuli across Testing block and Stimulus Condition in Experiment 3. All infants are reported in Figure $7 \mathrm{a}(\mathrm{N}=78)$ and only infants who completed all six blocks are reported in Figure $7 \mathrm{~b}(\mathrm{~N}=26)$. Error bars represented Standard Errors.

J Exp Child Psychol. Author manuscript; available in PMC 2020 February 01. 
Table 1:

Mean Proportions of Hits and False Alarms (FA) across Experiments and Conditions.

\begin{tabular}{lcc}
\hline Experiment/Condition & Hits & False Alarms \\
\hline Experiment 1A & & \\
Unimodal Auditory & 1.00 & 0.00 \\
Cross-modal Auditory & 1.00 & 0.13 \\
Unimodal Visual & 0.99 & 0.04 \\
Cross-modal Visual & 1.00 & 0.61 \\
Experiment 1B & & \\
Unimodal Auditory & 0.99 & 0.02 \\
Cross-modal Auditory & 0.97 & 0.02 \\
Unimodal Visual & 0.99 & 0.06 \\
Cross-modal Visual & 0.98 & 0.01 \\
Experiment 2A \& 2B & & \\
Unimodal Visual (2A) & 0.92 & 0.43 \\
Words at Test (2A) & 0.89 & 0.76 \\
Words absent at Test (2B) & 0.74 & 0.28 \\
\hline
\end{tabular}

